\title{
A SPECTRAL LINE SURVEY OF NGC 7027 AT MILLIMETER WAVELENGTHS
}

\author{
Yong ZHang AND Sun KwOK \\ Department of Physics, University of Hong Kong, Pokfulam Road, Hong Kong; zhangy96@hku.hk, sunkwok@hku.hk \\ AND \\ Dinh-V-TRUnG \\ Institute of Astronomy and Astrophysics, Academia Sinica, P.O. Box 23-141, Taipei 106, Taiwan; trung@asiaa.sinica.edu.tw \\ Received 2007 November 5; accepted 2008 January 6
}

\begin{abstract}
We report on a recent spectral line survey of the planetary nebula (PN) NGC 7027 using the Arizona Radio Observatory (ARO) $12 \mathrm{~m}$ telescope and the Heinrich Hertz Submillimeter Telescope (SMT) at millimeter wavelengths. The spectra covering the frequency ranges 71-111, 157-161, and 218-267 GHz were obtained with a typical sensitivity of rms $<8 \mathrm{mK}$. A total of 67 spectral lines are detected, 21 of which are identified with 8 molecular species, 32 with recombination lines from hydrogen and helium, and 14 remain unidentified. As the widths of emission lines from $\mathrm{CO}$, other neutral molecules, molecular ions, and recombination of $\mathrm{H}^{+}$and $\mathrm{He}^{+}$are found to be different from each other, the line strengths and profiles are used to investigate the physical conditions and chemical processes of the neutral envelope of NGC 7027. The column densities and fractional abundances relative to the $\mathrm{H}_{2}$ of the observed molecular species are calculated and compared with predictions from chemical models. We found evidence for overabundance of $\mathrm{N}_{2} \mathrm{H}^{+}$and underabundance of CS and $\mathrm{HNC}$ in NGC 7027, suggesting that X-ray emission and shock waves may play an important role in the chemistry of the hot molecular envelope of the young PN.
\end{abstract}

Subject headings: ISM: abundances — ISM: molecules — planetary nebulae: individual (NGC 7027) — radio lines: ISM

Online material: extended color figures

\section{INTRODUCTION}

We now realize that the circumstellar envelopes of evolved stars are active sites of molecular synthesis. Millimeter-wave spectroscopy has identified over 60 molecular species in the envelopes of asymptotic giant branch (AGB) stars, their descendant planetary nebulae (PNs), and the transition objects between the two phases, protoplanetary nebulae (PPNs; Olofsson 1997; Cernicharo et al. 2000; Ziurys et al. 2007). These gas-phase molecules are believed to be the precursors of complex organic compounds that are found in PPNs and PNs (Cernicharo et al. 2001a, 2001b; Kwok 2004). Since the molecular synthesis takes place in the circumstellar envelope through either gas-phase or grain-surface reactions, the study of the changing chemical composition and molecular abundance between objects in consecutive phases of evolution will provide useful information on the chemical pathways of their formation. Furthermore, since the circumstellar envelopes are expanding, the dynamical timescales impose a time limit on the reaction timescales. The typical dynamical timescales for AGB stars, PPNs, and PNs are $10^{4}-10^{5} \mathrm{yr}, 10^{3} \mathrm{yr}$, and $10^{3}-10^{4} \mathrm{yr}$, respectively. The observation of a specific molecular species in the PPN phase which is not seen in AGB stars implies that this molecule must be formed on timescales shorter than a few thousand years. The changing relative abundance of molecular species between different phases can also point to specific chemical pathways. Motivated by these considerations, we have performed spectral line surveys of several objects in the late stages of stellar evolution and try to provide a systematic and uniform study of circumstellar chemistry.

The study of the circumstellar chemistry of PNs is particularly interesting and challenging because of the additional dimension of photochemistry. Since part of the circumstellar envelope is ionized by ultraviolet radiation from the hot central star, mole- cules can be photoionized or photodissociated. The molecular composition of the circumstellar envelopes is therefore expected to be qualitatively different from AGB stars and PPNs. The young, carbon-rich PN NGC 7027 is known to be the PN with the richest molecular content. Because of its high mass $\left(0.7 M_{\odot}\right)$, the central star of NGC 7027 is evolving very quickly, reaching an effective temperature of $219,000 \mathrm{~K}$ a few hundred years after the onset of photoionization (Zhang et al. 2005). The remnant of the AGB envelope is eroded by a nascent ionized region of $10^{\prime \prime}$. Imaging observations have shown that a thin photodissociation region (PDR) separates the ionized region and the extended molecular envelope with a size $>70^{\prime \prime}$ (see Hasegawa \& Kwok 2001 and the references therein). A large number of molecular emission lines have been detected by infrared (IR) and radio spectroscopy (e.g., Liu et al. 1996; Cox et al. 1993; Hasegawa \& Kwok 2001; Herpin et al. 2002). Unambiguously detected molecular species in NGC 7027 include $\mathrm{H}_{2}, \mathrm{OH}, \mathrm{CO}, \mathrm{CO}^{+}, \mathrm{CH}, \mathrm{CH}^{+}, \mathrm{HCN}, \mathrm{HNC}, \mathrm{HCO}^{+}$, $\mathrm{H}_{2} \mathrm{O}, \mathrm{N}_{2} \mathrm{H}^{+}, \mathrm{CN}, \mathrm{CS}, \mathrm{C}_{2} \mathrm{H}, \mathrm{C}_{3} \mathrm{H}_{2}, \mathrm{SiS}$, and their isotopomers. A systematic molecular-line observation of PNs by Bachiller et al. (1997) and Josselin \& Bachiller (2003) has found that the column densities of molecules observed in NGC 7027 are generally larger than those in other PNs. Compared with other PNs, NGC 7027 shows a low $\mathrm{HNC}$ abundance and an abnormally high $\mathrm{N}_{2} \mathrm{H}^{+}$ (Bachiller et al. 1997). Hasegawa \& Kwok (2001) suggested that most of the observed molecular lines are emitted from the PDR of the object, except for $\mathrm{CO}$ emission, which arises from a much more extended region. According to Cox et al. (1987), lines from the ring molecule $\mathrm{C}_{3} \mathrm{H}_{2}$ are emitted from dense regions. The observations by Thronson \& Bally (1986) and Deguchi et al. (1990) suggested that grain chemistry probably plays an important role for the formation of molecules in NGC 7027.

The chemistry in the PDR of NGC 7027 has been modeled by Yan et al. (1999), who suggested that the molecular abundance is 

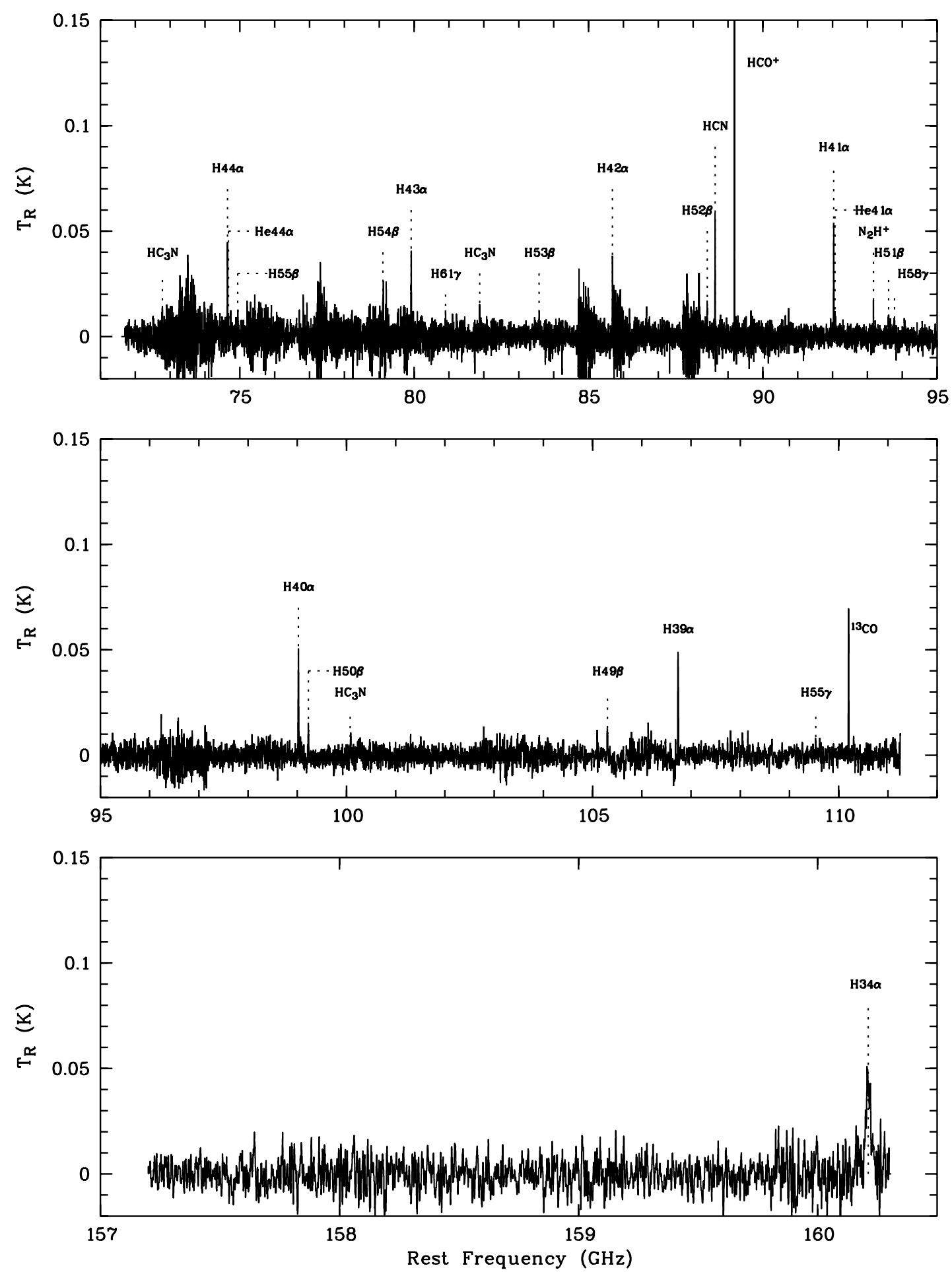

FIG. 1.-From top to bottom: 71-111, 157-161, and 157-161 GHz MAC spectra of NGC 7027 obtained with the ARO $12 \mathrm{~m}$ telescope. The spectra have been smoothed to a resolution of $1 \mathrm{MHz}$. The spectra at full resolution are available in Fig. 3.

significantly influenced by both photodissociation and shock chemistry. A detailed chemical model of the neutral envelope of NGC 7027 by Hasegawa et al. (2000) suggested that most of the detected molecules in NGC 7027 are formed over a short timescale after the object evolved into the PN stage. This was supported by the observations of Herpin et al. (2002) with the Infrared Space Observatory (ISO). However, a different conclusion was obtained by Josselin \& Bachiller (2003), who suggested that most of molecules can survive during the transition from AGB to PN. Redman et al. (2003) modeled the chemical evolution from AGB to PPN and
PN considering the effect of clumpiness. By comparing model results and observations, they proposed that the clumps form at an early stage of PN and may be plentifully present within NGC 7027.

Hydrogen and helium recombination lines from NGC 7027 are detectable at millimeter wavelengths, allowing us to investigate the physical conditions and dynamics of the ionized regions. An advantage of the radio data is the reduced effect of dust extinction. A few radio recombination lines from $\mathrm{H} 56 \alpha-\mathrm{H} 110 \alpha$ have been detected in NGC 7027 (Roelfsema et al. 1991; Chaisson \& Malkan 1976; Ershov \& Berulis 1989 and references therein). 


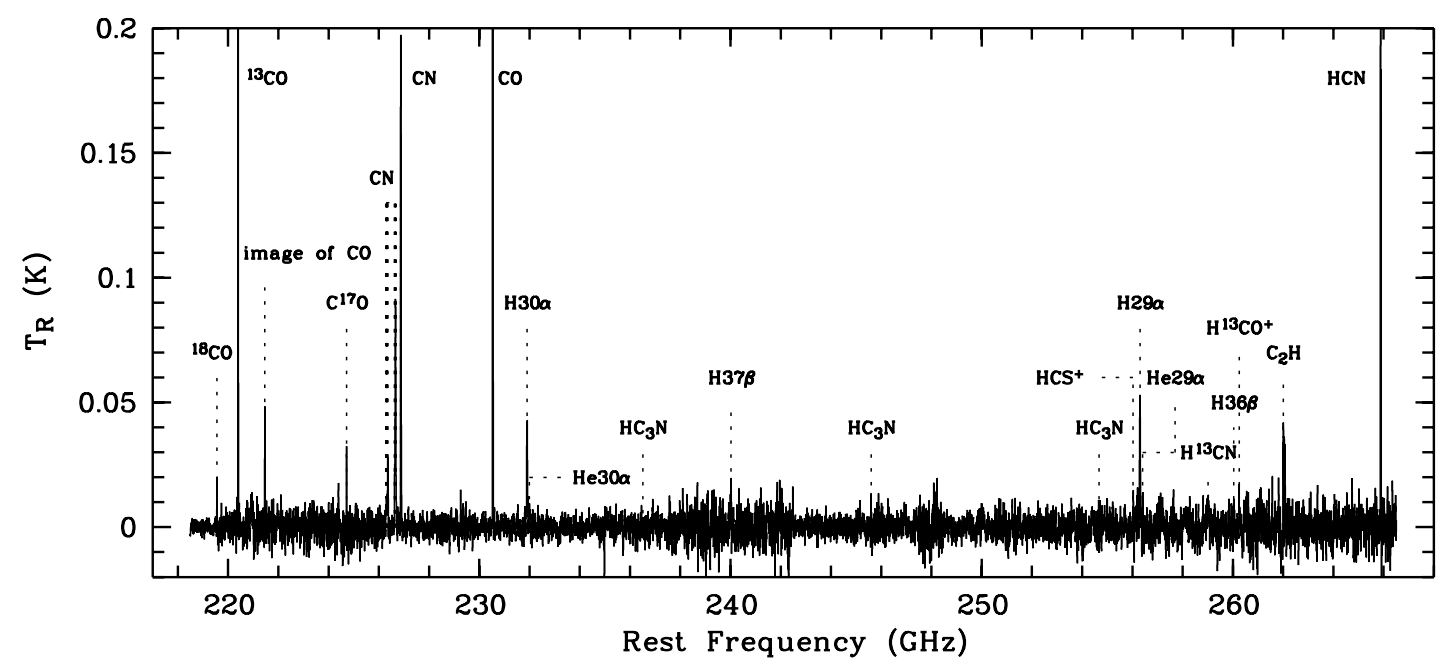

Fig. 2.- The 218-267 GHz FFB spectrum of NGC 7027 obtained with the SMT $10 \mathrm{~m}$ telescope. The spectra have been smoothed to a resolution of $3 \mathrm{MHz}$. The spectra at full resolution are available in Fig. 4.

Ershov \& Berulis (1989) constructed a dynamical model to explain the observed radio recombination lines and found that the expansion velocity of the ionized gas is in good agreement with that of the molecular envelope. Roelfsema et al. (1991) observed the H76 $\alpha$ line and argued that the ionized gas is decelerated by the surrounding molecular material.

In this paper, we report a systematic spectral survey of NGC 7027 using the ARO $12 \mathrm{~m}$ and the SMT $10 \mathrm{~m}$ telescopes, ${ }^{1}$ covering the frequency ranges $71-111,157-161$, and $218-267 \mathrm{GHz}$. These data allow us to investigate the physical conditions and chemical compositions of the molecular envelope of this PN. This paper is organized as follows: $\S 2$ describes the observations and the procedures of data reduction; $\S 3$ presents the identification and measurements of detected emission lines; in $\S 4$ the method of data analysis is described and the resulting excitation temperatures, column densities, and abundances relative to $\mathrm{H}_{2}$ are presented; in $\S 5$, we discuss the physical and chemical characteristics in the molecular envelope and ionized regions; and the main conclusions are summarized in $\S 6$.

\section{OBSERVATIONS AND DATA REDUCTION}

The spectral survey was carried out between 2005 April and 2006 September. The observations were made in beam-switching mode with an azimuth beam throw of $2^{\prime}$. Pointing accuracy was checked every $2 \mathrm{hr}$. The on-source integration time was more than $1 \mathrm{hr}$ for each frequency setting.

The 71-111 and $157-161 \mathrm{GHz}$ spectra were obtained using the ARO $12 \mathrm{~m}$ telescope at Kitt Peak. The dual-channel SIS receivers were employed in single-sideband mode, yielding a system temperature of $150-400 \mathrm{~K}$. The image rejection ratio was $16-20 \mathrm{~dB}$. The receiver back ends were equipped with two 256 channel filter banks (FBs) with spectral resolutions of $500 \mathrm{kHz}$ and $1 \mathrm{MHz}$ and a millimeter autocorrelator (MAC) with 3072 channels and $195 \mathrm{kHz}$ resolution. All spectrometers were operated in series mode. Two orthogonal linear polarization modes were measured. The temperature scale, $T_{R}^{*}$, was calibrated and corrected for atmospheric attenuation, radiative loss, and rearward and forward scattering and spillover by the chopper-wheel

\footnotetext{
${ }^{1}$ The $12 \mathrm{~m}$ telescope and the Heinrich Hertz Submillimeter Telescope (SMT) is operated by the Arizona Radio Observatory (ARO), Steward Observatory, University of Arizona.
}

method. The main-beam brightness temperature was derived using $T_{R}=T_{R}^{*} / \eta_{m}^{*}$, where $\eta_{m}^{*}$ is the corrected beam efficiency. Over the frequency range $71-161 \mathrm{GHz}, \eta_{m}^{*}$ is from 0.94 to 0.71 , and the conversion factor is $32.5-29.1 \mathrm{Jy} \mathrm{K}^{-1}$.

The 218-267 GHz observations were carried out with the SMT $10 \mathrm{~m}$ telescopes at Mount Graham, Arizona, using the dualchannel SIS receivers operated in single-sideband dual polarization mode. The system temperature is typically $400-700 \mathrm{~K}$. The spectrometers used were a 2048 channel acousto-optical spectrometer (AOS) with a spectral resolution of $500 \mathrm{kHz}$ per channel and 1024 channel Forbes Filterbanks (FFBs) with a spectral resolution of $1 \mathrm{MHz}$ per channel. All spectrometers were used simultaneously. The data were calibrated to the antenna temperature scale, $T_{A}^{*}$, which was corrected for atmospheric attenuation. $T_{A}^{*}$ is converted to main-beam temperature by $T_{R}=T_{A}^{*} / \eta_{\mathrm{mb}}$, where the main-beam efficiency, $\eta_{\mathrm{mb}}$, is $\sim 0.7$. The conversion factor is $35 \mathrm{Jy} \mathrm{K}^{-1}$.

All spectra were reduced using the CLASS software package in GILDAS. ${ }^{2}$ After discarding the bad scans, which are seriously affected by bandpass irregularities, we co-added the calibrated spectral data using the rms noise of each spectrum as weights. A low-order polynomial baseline defined by the line-free spectral regions was subtracted from each spectrum. In order to improve the signal-to-noise ratio, the ARO $12 \mathrm{~m}$ and SMT $10 \mathrm{~m}$ spectra were smoothed and rebinned to a spectral resolution of 1 and $3 \mathrm{MHz}$, respectively, yielding a typical rms noise temperature of $<8 \mathrm{mK}$ in main-beam temperature unit. Based on a few strong lines detected in different spectrometers and different epochs, we estimate that the calibration uncertainties introduced by instruments and atmospheric conditions amount to about $15 \%$.

\section{THE SPECTRA}

The spectra of NGC 7027 obtained with the ARO $12 \mathrm{~m}$ telescope and the SMT $10 \mathrm{~m}$ telescope are presented in Figures 1 and 2, respectively. The spectra at full resolution are shown in Figures 3 and 4 , which are available in their entirety in the electronic version of this manuscript. Note that some features in these figures are caused by bandpass irregularities. More molecular lines were detected in the $10 \mathrm{~m}$ spectra compared to the $12 \mathrm{~m}$ spectra, as the ARO $12 \mathrm{~m}$ telescope has a larger beam size and thus bears larger

\footnotetext{
${ }^{2}$ GILDAS is developed and distributed by the Observatorie de Grenoble and IRAM
} 

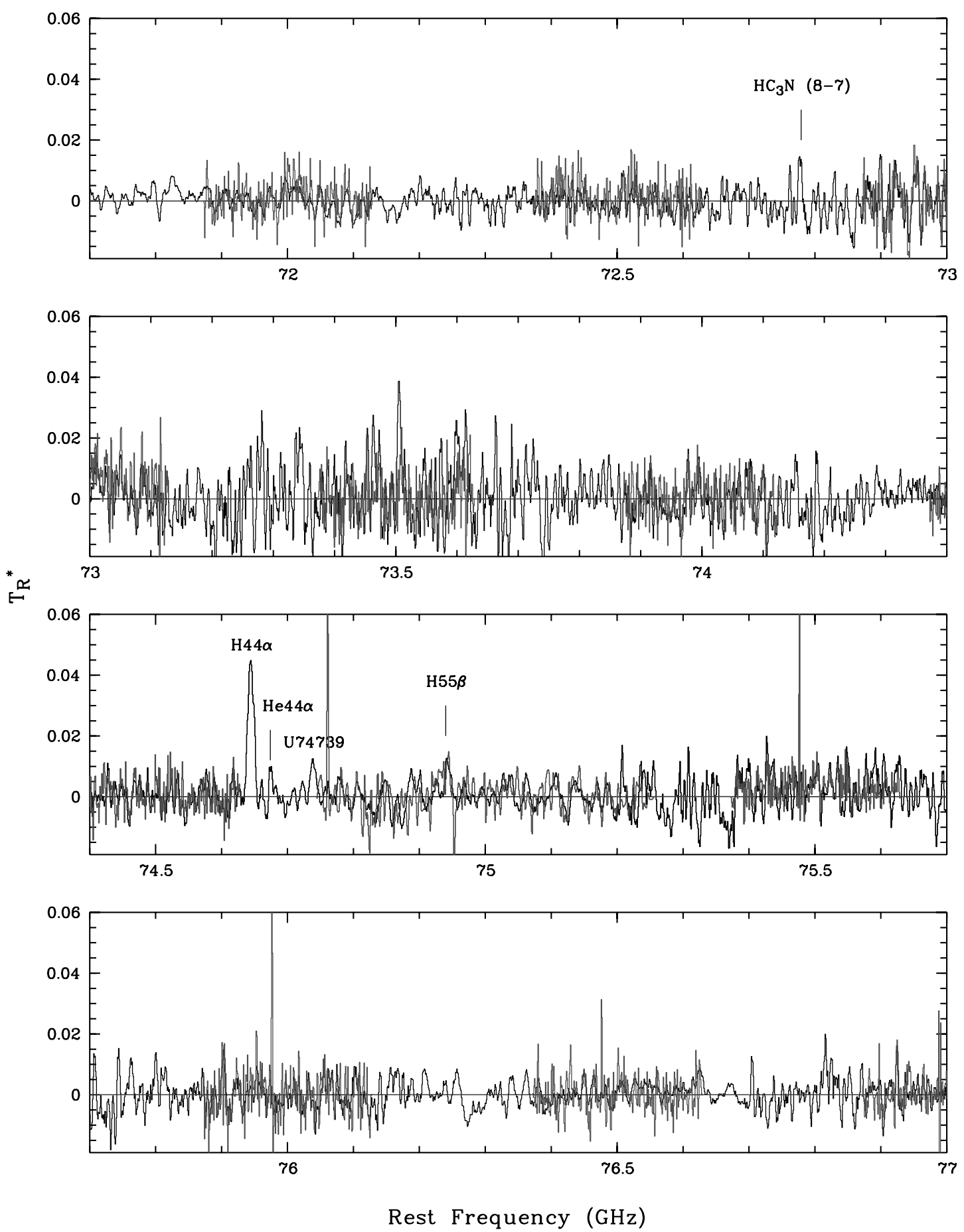

FIG. 3.-The 71-111 and 157-161 GHz spectra of NGC 7027 obtained with the ARO $12 \mathrm{~m}$ telescope. The black and gray lines are the MAC and FB data, respectively. The spectral resolution is $1 \mathrm{MHz}$. The colon represents uncertain detection. [See the electronic edition of the Journal for an extended color version of this figure.]

beam dilution effect. Sections of the spectra containing spectral lines are expanded in Figures $5(12 \mathrm{~m})$ and $6(10 \mathrm{~m})$ in order to show the line profiles. Since each spectrum was simultaneously observed with two receiver back ends, an emission line should be recorded by both spectrometers. This allows us to distinguish between real emission lines and artificial features. Contamination from image band was carefully checked. The feature at $221.463 \mathrm{GHz}$ was found to be the $\mathrm{CO}(2-1)$ line from the image band. No other lines from the image band were apparent. A total of 71 distinct emission features were measured.
The molecular lines are identified on the basis of the JPL catalog (Pickett et al. 1998) ${ }^{3}$ and the Cologne database for molecular spectroscopy (CDMS; Müller et al. 2001, 2005). ${ }^{4}$ The suggested identifications are labeled in Figures 1 and 2. Combining the single and blended features, we identified 21 molecular lines, belonging to eight molecular species $\left(\mathrm{CO}, \mathrm{CN}, \mathrm{C}_{2} \mathrm{H}, \mathrm{HCN}, \mathrm{HCO}^{+}, \mathrm{HCS}^{+}\right.$, $\mathrm{HC}_{3} \mathrm{~N}, \mathrm{~N}_{2} \mathrm{H}^{+}$) with a total of 13 isotopomers. We also present

\footnotetext{
3 See http://spec.jpl.nasa.gov.

4 See http://www.ph1.uni-koeln.de/vorhersagen.
} 

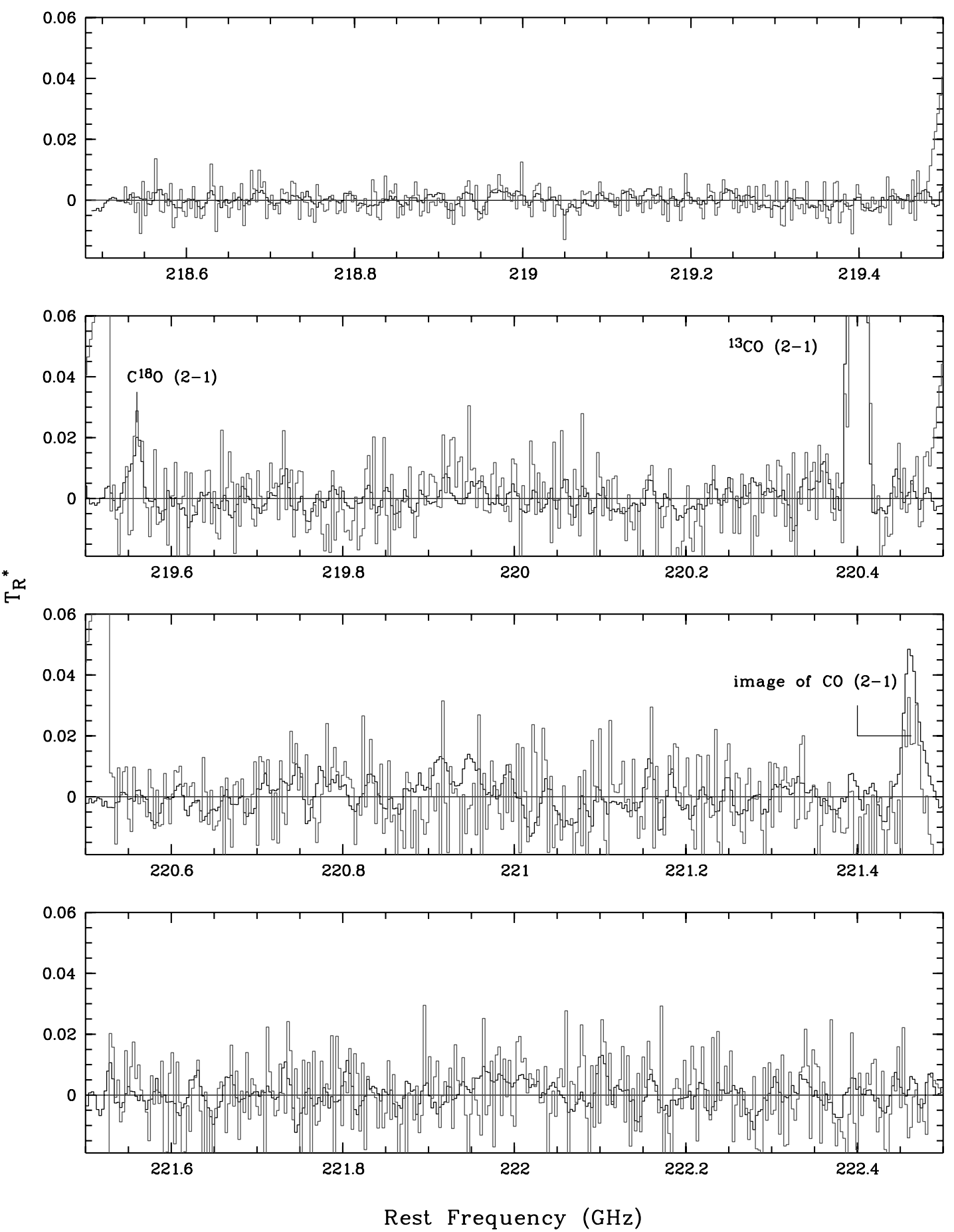

FIG. 4.- The 218-267 GHz spectrum of NGC 7027 obtained with the SMT $10 \mathrm{~m}$ telescope. The black and gray lines are the FFB and AOS data, respectively. The spectral resolution is $3 \mathrm{MHz}$. The colon represents uncertain detection. [See the electronic edition of the Journal for an extended color version of this figure.]

possible detection of $\mathrm{C}_{3} \mathrm{H}_{2}, \mathrm{HC}^{17} \mathrm{O}^{+}$, and $\mathrm{HC}^{18} \mathrm{O}^{+}$. Fourteen lines remain unidentified. Several of these transitions are discovered for the first time in NGC 7027. A list of molecular lines detected in our spectra is presented in Table 1. The table also gives the rms noise, the main-beam temperatures, and the integrated intensities (in units of $\mathrm{K} \mathrm{km} \mathrm{s}^{-1}$ ) of the lines.

The $\mathrm{CO}(2-1)$ and ${ }^{13} \mathrm{CO}(1-0,2-1)$ lines have been extensively observed by various telescopes in the past (see, e.g., Hasegawa $\&$ Kwok 2001). The integrated intensities of the $\mathrm{CO}(2-1)$ and ${ }^{13} \mathrm{CO}(2-1)$ lines presented in Table 1 are lower than those reported by Hasegawa \& Kwok (2001; see their Table 1). However, we note that the $\mathrm{CO}(2-1) /{ }^{13} \mathrm{CO}(2-1)$ integrated intensity ratio of
24.8 is in excellent agreement the value of 21.8 in Hasegawa \& Kwok (2001). The lower antenna temperatures detected here are due to the larger beam dilution effect since the beam size of the SMT $10 \mathrm{~m}$ telescope is a factor of $\sim 2$ larger than the $15 \mathrm{~m}$ James Clarke Maxwell Telescope used by Hasegawa \& Kwok (2001). The $\mathrm{C}^{18} \mathrm{O}(2-1)$ and $\mathrm{C}^{17} \mathrm{O}(2-1)$ lines were clearly detected in our spectra. To our knowledge, it is the first time that these two transitions have been detected in NGC 7027.

Three strong $\mathrm{CN}(2-1)$ hyperfine groups are detected in our observations. These lines have previously been seen by Cox et al. (1993), Josselin \& Bachiller (2003), and Bachiller et al. (1997). The emission feature at $227.2 \mathrm{GHz}$ is only marginally above our 


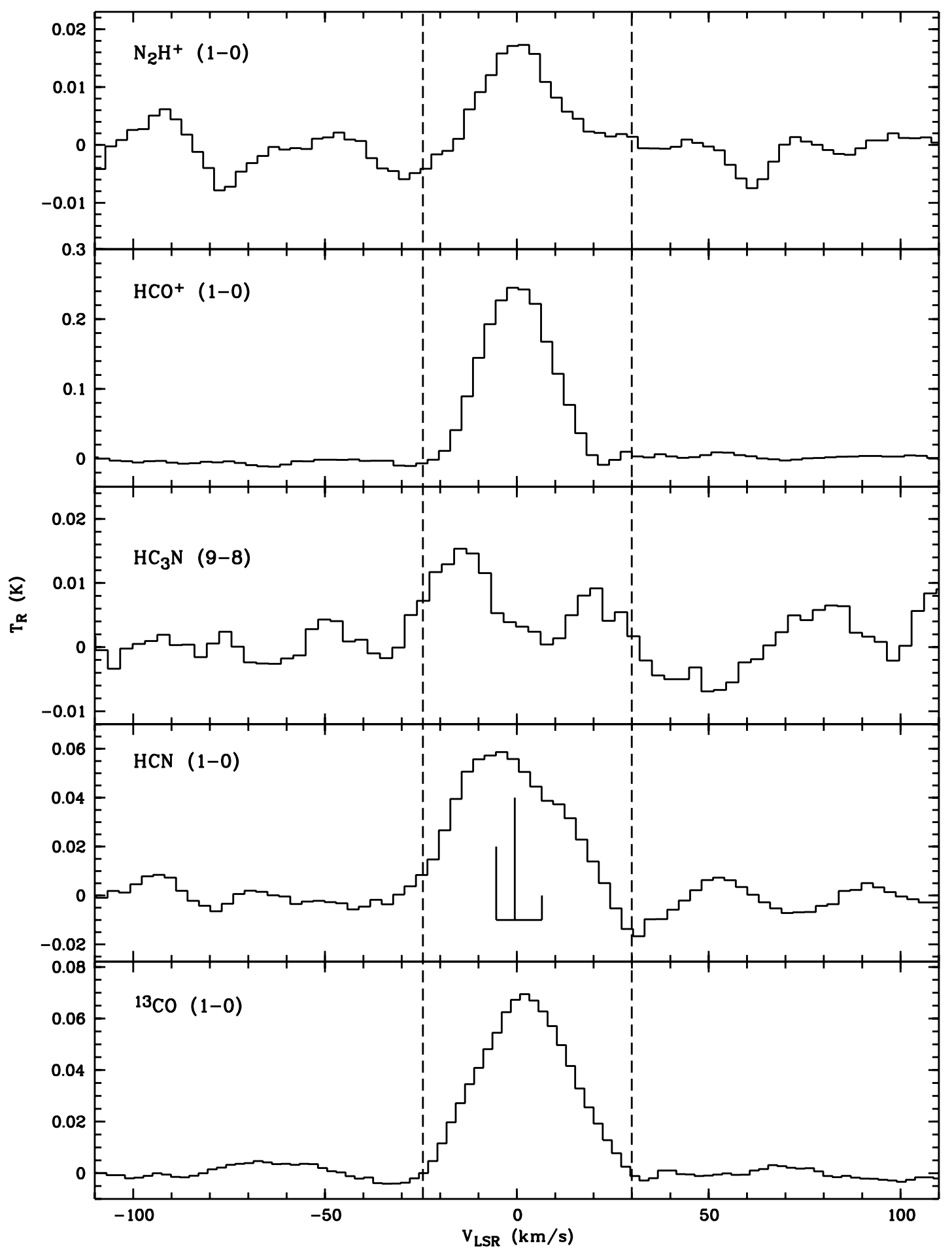

FIG. 5.- Line profiles of NGC 7027 observed by the ARO $12 \mathrm{~m}$ telescope. Positions and relative intensities of hyperfine components are marked by vertical solid lines. The spectral resolution is $1 \mathrm{MHz}$. The velocity range of ${ }^{13} \mathrm{CO}$ emission (above $1 \sigma$ ) is indicated by vertical dashed lines.

limit of detection and may be a combination of the $\mathrm{CN}(N=2-1$, $J=5 / 2-1 / 2)$ and the $\mathrm{C}_{3} \mathrm{H}_{2}\left(4_{32}-3_{21}\right)$ transitions. Other previous detections of CN in NGC 7027 include the $J=3-2$ and $J=1-0$ transitions at 340 and $114 \mathrm{GHz}$, respectively (Hasegawa \& Kwok 2001; Bachiller et al. 1997; Thronson \& Bally 1986; Cox et al. 1993; Josselin \& Bachiller 2003).

The $\mathrm{C}_{2} \mathrm{H}(3-2)$ transitions in NGC 7027, first discovered by Hasegawa \& Kwok (2001), are confirmed in our observations. All the $\mathrm{C}_{2} \mathrm{H}(3-2)$ lines show a double-peaked profile. The $\mathrm{C}_{3} \mathrm{H}_{2}\left(2_{20}-\right.$ $\left.2_{11}\right)$ at $18.3 \mathrm{GHz}$ and the $\mathrm{C}_{3} \mathrm{H}_{2}\left(1_{10}-1_{01}\right)$ at $21.6 \mathrm{GHz}$ transitions were first detected in the PN by Cox et al. (1987). We attempted to search for two new $\mathrm{C}_{3} \mathrm{H}_{2}$ transitions $\left(6_{25}-5_{14}\right.$ and $\left.5_{33}-4_{22}\right)$ at higher frequencies. The two lines are extremely faint, with intensities between 2 and $3 \sigma$ noise level. Hence, our detection of $\mathrm{C}_{3} \mathrm{H}_{2}$ should be treated with some caution.

The well-studied $\mathrm{HCN}(1-0,3-2)$ and $\mathrm{HCO}^{+}(3-2)$ transitions are also detected. We also detected their isotopic transitions $\mathrm{H}^{13} \mathrm{CN}(3-2)$ and $\mathrm{H}^{13} \mathrm{CO}^{+}(3-2)$. However, the transitions $\mathrm{H}^{13} \mathrm{CN}(1-0)$ at $86.340 \mathrm{GHz}$ and $\mathrm{H}^{13} \mathrm{CO}^{+}(1-0)$ at $86.754 \mathrm{GHz}$ are overwhelmed by noise. The transitions $\mathrm{HC}^{17} \mathrm{O}^{+}(1-0)$ and 


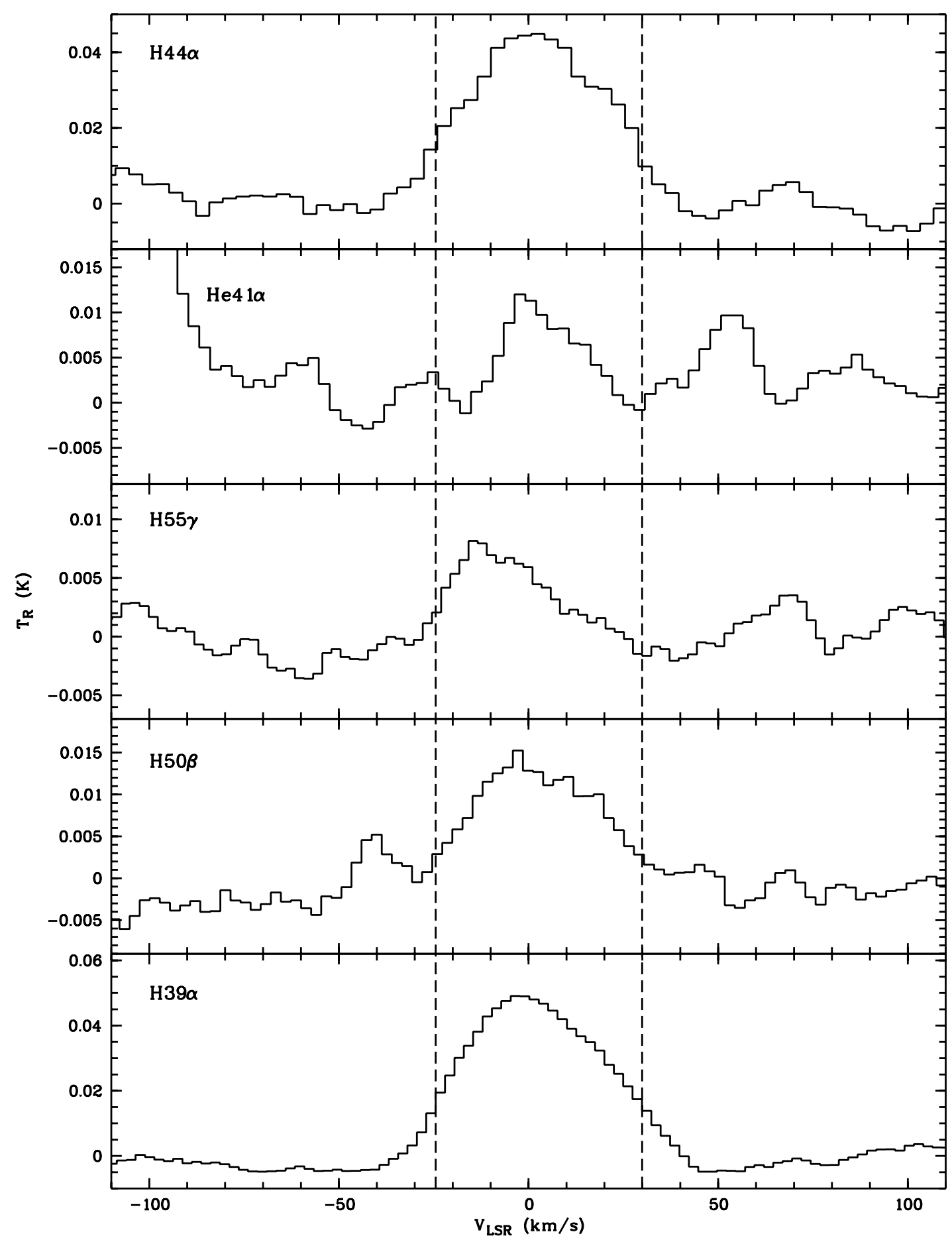

FIG. 5-Continued

$\mathrm{HC}^{18} \mathrm{O}^{+}(1-0)$ are only marginally above $2 \sigma$ noise level, and thus have an ambiguous detection. A narrow feature at $256.028 \mathrm{GHz}$ has been discovered and identified with the $\operatorname{HCS}^{+}(6-5)$ transition, confirming the observations by Hasegawa \& Kwok (2001). We also detect the $\mathrm{N}_{2} \mathrm{H}^{+}(1-0)$ line which was first reported by Cox et al. (1993).

An unsuccessful search for the $\mathrm{HC}_{3} \mathrm{~N}(11-10)$ transition at 100.076 GHz was made by Thronson \& Bally (1986). ${ }^{5}$ In our ob-

\footnotetext{
${ }^{5}$ Note that in Table 1 of Thronson \& Bally (1986) the frequency of the $\mathrm{HC}_{3} \mathrm{~N}(11-10)$ transition is given as " $110.0 \mathrm{GHz}$ " by mistake.
}

servations, several $\mathrm{HC}_{3} \mathrm{~N}$ lines are identified in the spectra from both the ARO $12 \mathrm{~m}$ telescope and the SMT $10 \mathrm{~m}$ telescope. However, we do not detect the transitions $\mathrm{HC}_{3} \mathrm{~N}(10-9)$ at $90.979 \mathrm{GHz}$ and $\mathrm{HC}_{3} \mathrm{~N}(12-11)$ at $109.174 \mathrm{GHz}$, making our identification arguable. If confirmed, this is the first detection of this species in a PN.

The unidentified lines are listed in Table 2. Most of them are very faint, and are only marginally detected. Some of these lines have been detected in the star formation regions Sgr B2(N) and Sgr B2(M) (Turner 1989; Nummelin et al. 1998). We also compared our spectra with a recent molecular line survey of the PPN CRL 618 by Pardo et al. (2007). These $U$ lines are not detected in 


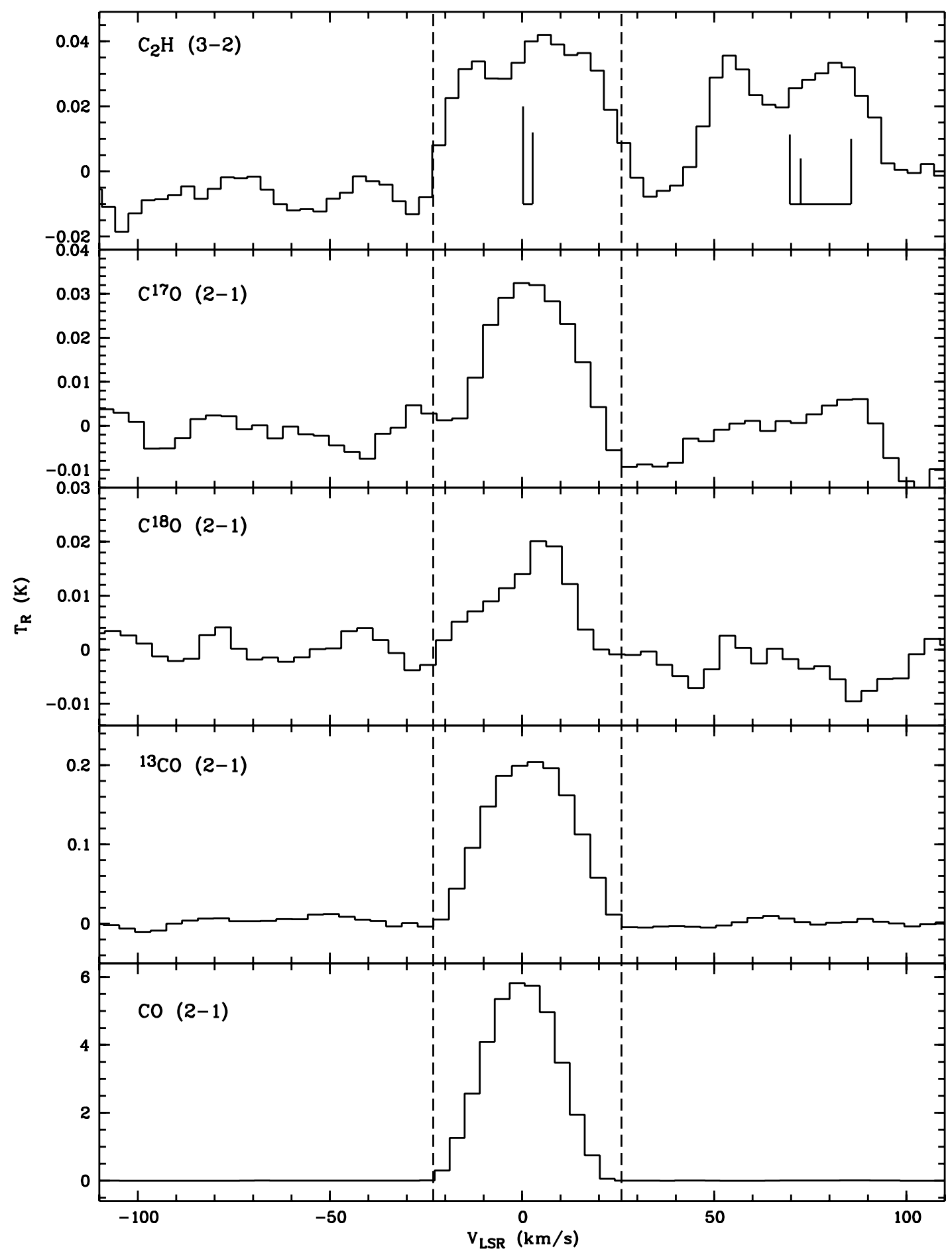

FIG. 6. - Line profiles of NGC 7027 observed by the SMT $10 \mathrm{~m}$ telescope. The description is the same as in Fig. 5. The spectral resolution is $3 \mathrm{MHz}$.

their spectra. Thus we infer that some of these lines probably arise from species that are formed in very strong UV radiation.

Table 3 lists a number of molecular species that are not detected by our observations of NGC 7027. For a given species, the strongest transition in our frequency ranges is given in the table aiming to reach the tightest constrain on its column density. The upper limits of the integrated intensities can be obtained by $3 \sigma \Delta v / \sqrt{n}$, where $\sigma$ is the rms noise, and $n$ is the number of channels within the line width $\Delta v$.

A number of recombination lines, including 24 hydrogen lines and eight helium lines, are detected in our observations. Pre- viously, most of the recombination lines were observed in the low-frequency bands. The identifications of recombination lines are based on the calculations by Lilley \& Palmer (1968) and Towle et al. (1996). No heavy-element recombination lines are visible in our spectra, as they are under our detection limit. The identifications and measurements of the recombination lines are presented in Table 4. All recombination lines were measured using Gaussian line profile fitting. Some of the lines, such as H36 $\beta$, are probably blended with unknown features and show a relatively broad profile. The $\mathrm{H} 60 \gamma$ line at $84.914 \mathrm{GHz}$, the $\mathrm{H} 60 \gamma$ line, and the $\mathrm{He} 42 \alpha$ line at $85.723 \mathrm{GHz}$ fall within the spectral regions that 


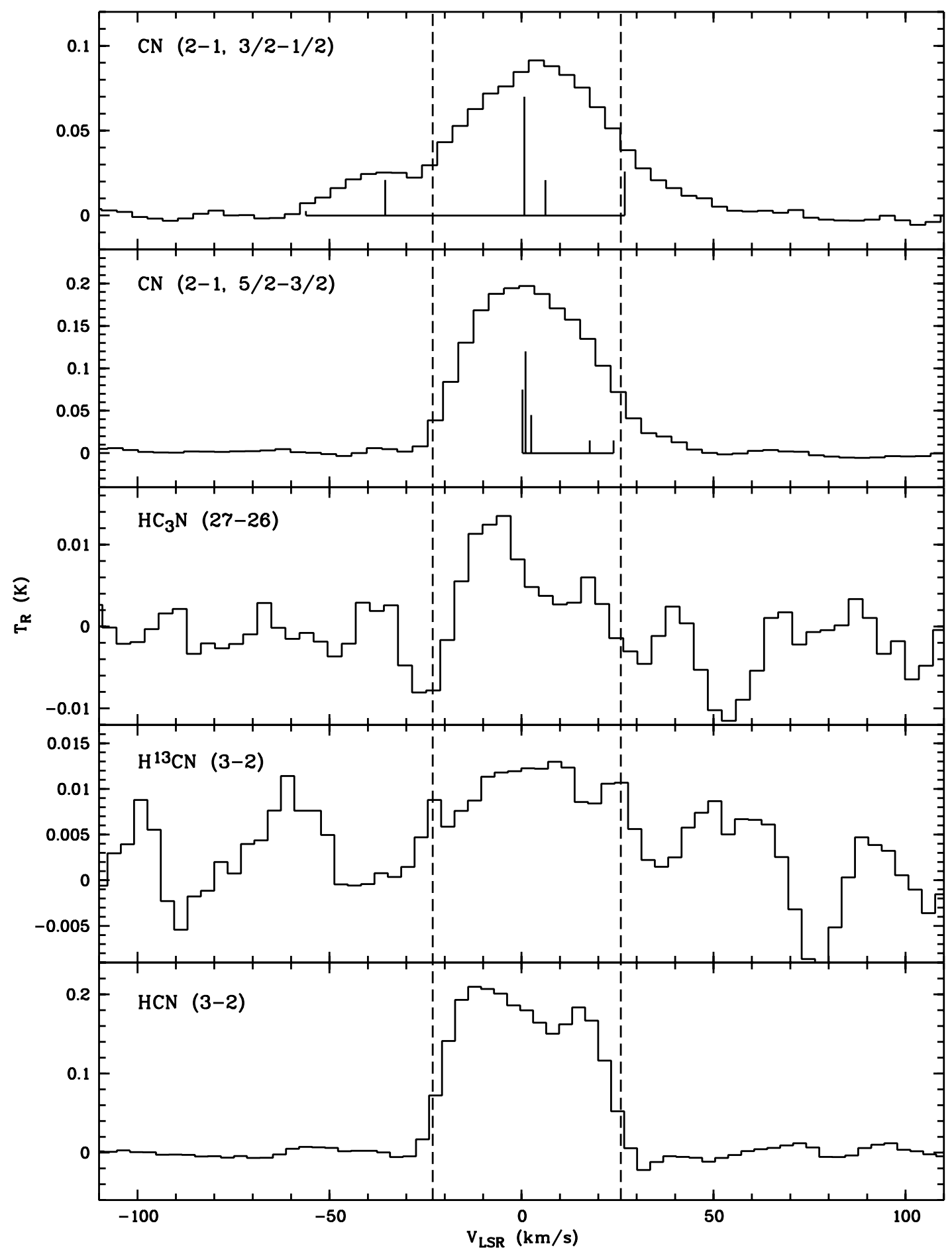

Fig. 6-Continued

have a low signal-to-noise ratios (see Fig. 1), and thus their measurements are uncertain.

\section{ANALYSIS}

\subsection{Excitation Temperatures and Column Densities}

In order to obtain molecular excitation temperatures and column densities, the effect of beam dilution is corrected under the assumption that both the source brightness distribution and the antenna beam have a Gaussian profile. The source brightness temperature (which is equivalent to source intensity) was obtained by
$T_{s}=T_{R}\left(\theta_{b}^{2}+\theta_{s}^{2}\right) / \theta_{s}^{2}$, where the main-beam brightness temperature $T_{R}$ has the physical meaning of average intensity, $\theta_{b}$ is the antenna full beam at half-power, and $\theta_{s}$ is the source diameter. The beam sizes of the ARO $12 \mathrm{~m}$ telescope and the SMT $10 \mathrm{~m}$ telescope are $86^{\prime \prime}-38^{\prime \prime}$ and $32^{\prime \prime}-28^{\prime \prime}$ over the frequency ranges $71-161$ and $218-268 \mathrm{GHz}$, respectively. Different molecular species in NGC 7027 might have different distributions and $\theta_{s}$, and thus should be separately estimated in terms of the map of each species. Based on the observations of Hasegawa \& Kwok (2001), we have assumed $\theta_{s}=13.2^{\prime \prime}$ for all the species except CO. Highresolution mapping observations of CO in NGC 7027 suggest that 


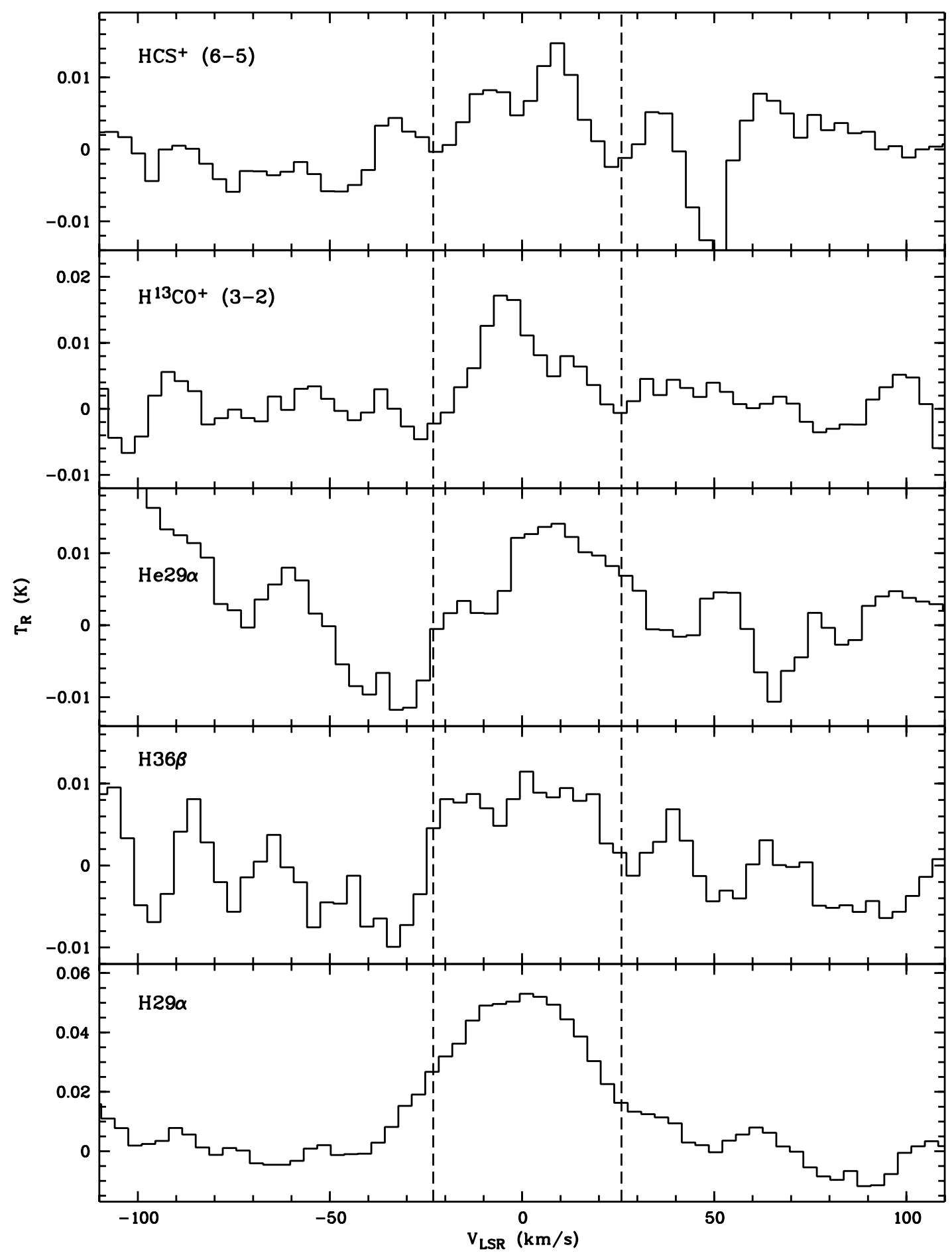

Fig. 6-Continued

the $\mathrm{CO}$ emission originates in a more extended region (Masson et al. 1985), so we adopted $\theta_{s}=40^{\prime \prime}$ for $\mathrm{CO}$ and its isotopomers.

The standard rotation-diagram method was applied to determine molecular excitation temperatures and column densities. Under the assumption that (1) the lines are optically thin; (2) the level populations are in LTE and can be described by a Boltzmann temperature, referred to as the excitation temperature $\left(T_{\mathrm{ex}}\right)$; and (3) $T_{\mathrm{ex}}$ is much larger than the background temperature $\left(T_{\mathrm{bg}}\right)$, we have the relation

$$
\ln \frac{N_{u}}{g_{u}}=\ln \frac{3 k \int T_{s} d v}{8 \pi^{3} \nu S \mu^{2}}=\ln \frac{N}{Q\left(T_{\mathrm{ex}}\right)}-\frac{E_{u}}{k T_{\mathrm{ex}}},
$$

where $N_{u}, g_{u}$, and $E_{u}$ are the population, degeneracy, and excitation energy of the upper level, $\int T_{s} d v$ is the integrated intensity of the source, $S$ the line strength, $\mu$ the dipole moment, $\nu$ the line frequency, $Q$ the rotation partition function, and $N$ the column density. If many transitions for an individual molecule are observed, a straight line can be fitted by plotting the integrated intensity versus upper level energy. Then $T_{\mathrm{ex}}$ and the total column density can be deduced from the slope $\left(-1 / T_{\mathrm{ex}}\right)$ and intercept $[\ln (N / Q)]$ of this line.

Albeit with some problems, we probably detect five $\mathrm{HC}_{3} \mathrm{~N}$ transitions. The excitation energies of the upper levels cover a large 
TABLE 1

Detected Molecular Transitions

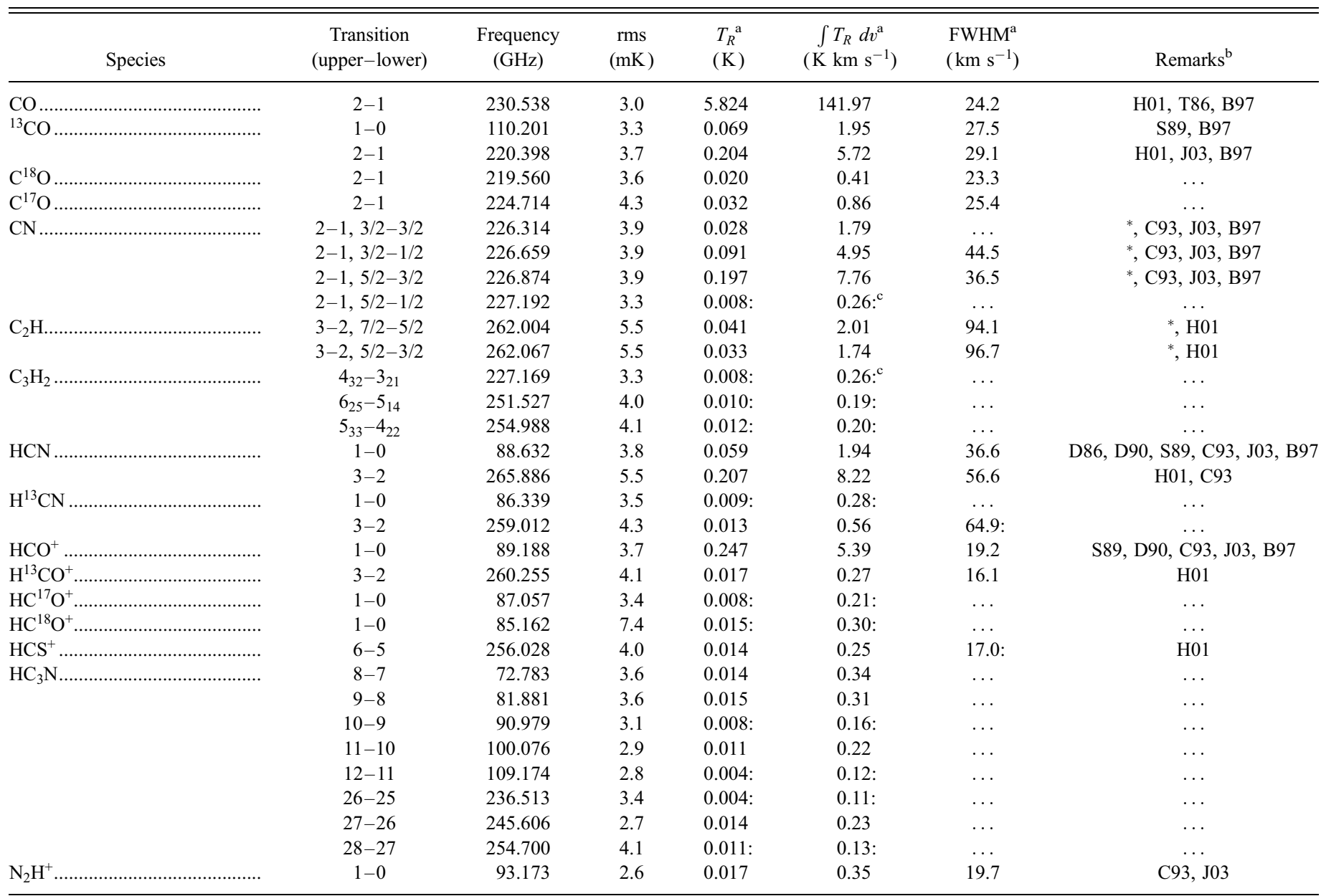

a The colon indicates uncertain detections.

b Also detected by Bachiller et al. (1997; B97); Cox et al. (1993; C93); Deguchi et al. (1986; D86); Deguchi et al. (1990; D90); Josselin \& Bachiller (2003; J03); Hasegawa \& Kwok (2001; H01); Sopka et al. (1989; S89); Thronson \& Bally (1986; T86). An asterisk represents unsolved fine-structure lines.

${ }^{c}$ The $\mathrm{CN}$ at $227.192 \mathrm{GHz}$ and the $\mathrm{C}_{3} \mathrm{H}_{2}$ at $227.169 \mathrm{GHz}$ lines are blended with each other.

TABLE 2

UnidenTIFIED FeATURES

\begin{tabular}{|c|c|c|c|}
\hline $\begin{array}{c}\text { Frequency } \\
(\mathrm{GHz})\end{array}$ & $\begin{array}{l}\mathrm{rms} \\
(\mathrm{mK})\end{array}$ & $\begin{array}{c}T_{R} \\
(\mathrm{~K})\end{array}$ & $\begin{array}{c}\int T_{R} d v \\
\left(\mathrm{~K} \mathrm{~km} \mathrm{~s}^{-1}\right)\end{array}$ \\
\hline $74.739 \ldots \ldots$ & 3.8 & 0.012 & 0.63 \\
\hline $89.656^{\mathrm{a}} \ldots \ldots \ldots \ldots \ldots \ldots$ & 3.6 & 0.012 & 0.26 \\
\hline $90.739^{\mathrm{a}}$ & 3.1 & 0.013 & 0.23 \\
\hline 95.246 & 3.2 & 0.011 & 0.47 \\
\hline $96.236^{\mathrm{a}}$ & 5.3 & 0.019 & 0.35 \\
\hline 98.079 & 2.9 & 0.010 & 0.20 \\
\hline 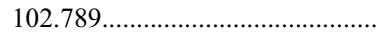 & 3.3 & 0.013 & 0.29 \\
\hline $107.802 .$. & 2.8 & 0.012 & 0.37 \\
\hline $229.256^{\mathrm{b}}$ & 3.7 & 0.015 & 0.22 \\
\hline $257.637^{\mathrm{b}}$ & 4.8 & 0.015 & 0.25 \\
\hline $261.569^{\mathrm{b}}$. & 5.5 & 0.020 & 0.28 \\
\hline $261.820 \ldots \ldots \ldots$ & 5.5 & 0.019 & 0.36 \\
\hline $263.369^{\mathrm{b}} \ldots \ldots \ldots \ldots$ & 5.0 & 0.017 & 0.26 \\
\hline 264.744 & 5.2 & 0.019 & 0.36 \\
\hline
\end{tabular}

${ }^{\text {a }}$ Also detected in Sagittarius B2 (M) (Turner 1989).

b Also detected in Sagittarius B2 (N) (Nummelin et al. 1998). range with $10 \mathrm{~K}<E / k<180 \mathrm{~K}$, allowing us to determine excitation temperature and column density. Figure 7 shows the rotation diagram for $\mathrm{HC}_{3} \mathrm{~N}$. A linear least-squares fit to all the transitions produces $N=4.39 \times 10^{13} \mathrm{~cm}^{-2}$. However, the data in the rotation diagram suggest that the low $E_{u}$ transitions seem to arise from a colder region. The results presented in Hasegawa et al. (2000) suggest that the neutral envelope of NGC 7027 consists of a geometrically thin and dense shell and an extended outer stellar wind region, with the temperature in the dense shell being more than a factor of 10 higher than that in the wind region. In this picture, our $\mathrm{HC}_{3} \mathrm{~N}$ high $E_{u}$ transitions would originate dominantly in the hot, dense shell, whereas the cold stellar wind region contributes significantly to the excitation of low $E_{u}$ lines. The stratified temperature structure may cause some problems in the calculations of column density. To get more precise results, detailed modeling is required, as done by Pardo et al. (2004) and Pardo \& Cernicharo (2007) for CRL 618. However, this is hampered by the weakness of most molecular lines in NGC 7027.

No rotation diagram is presented for other molecular species because either only one line for the species was detected, or the upper levels of the observed transitions have similar excitation energies. We thus adopted a constant excitation temperature of $T_{\text {ex }}=34.6 \mathrm{~K}$, as deduced from the $\mathrm{HC}_{3} \mathrm{~N}$ rotation diagram, and applied equation (1) to estimate the column densities of $\mathrm{CN}$, 
TABLE 3

Nondetected Molecular Species in NGC 7027

\begin{tabular}{|c|c|c|c|c|c|c|}
\hline \multirow[b]{2}{*}{ Species } & \multirow{2}{*}{$\begin{array}{c}\text { TRANSITION } \\
\text { (upper-lower) }\end{array}$} & \multirow{2}{*}{$\begin{array}{c}\text { FREQUENCY } \\
(\mathrm{GHz})\end{array}$} & \multirow{2}{*}{$\begin{array}{l}\mathrm{rms} \\
(\mathrm{mK})\end{array}$} & \multicolumn{2}{|c|}{$\begin{array}{c}N \\
\left(\mathrm{~cm}^{-2}\right)\end{array}$} & \multirow[b]{2}{*}{$N($ obs. $) / N(\text { model })^{2}$} \\
\hline & & & & Obs. $^{\mathrm{a}}$ & Model $^{\mathrm{b}}$ & \\
\hline 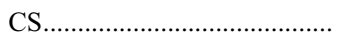 & $\nu=0 J=5-4$ & 244.936 & 2.7 & $1.9 \mathrm{E} 12$ & $2.5 \mathrm{E} 13$ & 0.08 \\
\hline $\mathrm{C}_{3} \mathrm{H} \ldots \ldots \ldots \ldots \ldots \ldots \ldots$ & ${ }^{2} \Pi_{3 / 2} J=23 / 2-21 / 2 \mathrm{~b}$ & 263.332 & 4.9 & $1.1 \mathrm{E} 13$ & $\ldots$ & $\ldots$ \\
\hline $\mathrm{C}_{4} \mathrm{H}$ & $N=24-23 \mathrm{a}$ & 228.349 & 2.9 & $1.7 \mathrm{E} 13$ & $1.4 \mathrm{E} 11$ & 121 \\
\hline $\mathrm{SiC}$ & ${ }^{3} \Pi_{2} J=6-5$ & 236.288 & 3.5 & $7.5 \mathrm{E} 12$ & $2.6 \mathrm{E} 12$ & 2.9 \\
\hline $\mathrm{SiO}$ & $\nu=0 J=6-5$ & 260.518 & 5.2 & $1.0 \mathrm{E} 13$ & $1.5 \mathrm{E} 13$ & 0.7 \\
\hline $\mathrm{SiS}$ & $\nu=0 J=14-13$ & 254.103 & 3.7 & $8.9 \mathrm{E} 13$ & $\ldots$ & $\ldots$ \\
\hline HNC & $J=1-0$ & 90.664 & 3.4 & $1.4 \mathrm{E} 12$ & $\ldots$ & $\ldots$ \\
\hline $\mathrm{HC}_{5} \mathrm{~N}$ & $J=27-26$ & 71.890 & 3.6 & $1.5 \mathrm{E} 12$ & $\ldots$ & $\ldots$ \\
\hline $\mathrm{CH}_{3} \mathrm{CN}$ & $J_{K}=5(1)-4(1), 5(0)-4(0)$ & 91.986 & 2.4 & $2.3 \mathrm{E} 13$ & $\ldots$ & $\ldots$ \\
\hline $\mathrm{H}_{2} \mathrm{CO}$ & $3(1,2)-2(1,1)$ & 225.678 & 3.9 & $4.5 \mathrm{E} 12$ & $9.2 \mathrm{E} 9$ & 489 \\
\hline 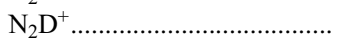 & $J=1-0$ & 77.109 & 4.1 & $2.8 \mathrm{E} 12$ & $\ldots$ & $\ldots$ \\
\hline
\end{tabular}

${ }^{\text {a }}$ Upper limits.

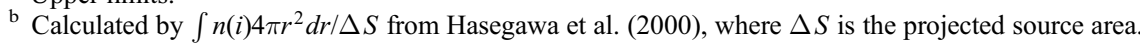

$\mathrm{C}_{2} \mathrm{H}, \mathrm{C}_{3} \mathrm{H}_{2}, \mathrm{HCS}^{+}, \mathrm{N}_{2} \mathrm{H}^{+}$, and $\mathrm{HCO}^{+}$. We derived a column density of $N=1.02 \times 10^{14} \mathrm{~cm}^{-2}$ for $\mathrm{CN}$, which is consistent with the values of $3.5 \times 10^{14} \mathrm{~cm}^{-2}$ and $6.1 \times 10^{13} \mathrm{~cm}^{-2}$ deduced by Hasegawa \& Kwok (2001) and Josselin \& Bachiller (2003), respectively. Our analysis yields $N=3.49 \times 10^{14} \mathrm{~cm}^{-2}$ for $\mathrm{C}_{2} \mathrm{H}$, in good agreement with the value of $2.4 \times 10^{14} \mathrm{~cm}^{-2}$ given by Hasegawa et al. (2000). From the observations of the $\mathrm{C}_{3} \mathrm{H}_{2}\left(1_{10}-1_{01}\right)$

TABLE 4

Recombination Lines IN NGC 7027

\begin{tabular}{|c|c|c|c|c|}
\hline Line & $\begin{array}{l}\text { Frequency } \\
\quad(\mathrm{GHz})\end{array}$ & $\begin{array}{c}T_{R} \\
(\mathrm{~K})\end{array}$ & $\begin{array}{c}\int T_{R} d v \\
\left(\mathrm{~K} \mathrm{~km} \mathrm{~s}^{-1}\right)\end{array}$ & $\begin{array}{c}\text { FWHM } \\
\left(\mathrm{km} \mathrm{s}^{-1}\right)\end{array}$ \\
\hline $\mathrm{H} 44 \alpha \ldots \ldots \ldots$ & 74.645 & 0.045 & 2.01 & 46.6 \\
\hline $\mathrm{H} 43 \alpha \ldots \ldots$ & 79.913 & 0.037 & 1.77 & 52.5 \\
\hline $\mathrm{H} 42 \alpha \ldots \ldots$ & 85.688 & 0.037 & 1.68 & 35.3 \\
\hline $\mathrm{H} 41 \alpha \ldots \ldots \ldots$ & 92.034 & 0.054 & 2.39 & 41.2 \\
\hline $\mathrm{H} 40 \alpha \ldots \ldots \ldots$ & 99.022 & 0.051 & 2.19 & 43.5 \\
\hline $\mathrm{H} 39 \alpha \ldots \ldots .$. & 106.737 & 0.049 & 2.47 & 49.4 \\
\hline $\mathrm{H} 34 \alpha \ldots \ldots .$. & 160.212 & 0.048 & 2.77 & 40.6 \\
\hline $\mathrm{H} 30 \alpha \ldots \ldots$ & 231.901 & 0.037 & 1.88 & 46.2 \\
\hline $\mathrm{H} 29 \alpha \ldots \ldots .$. & 256.302 & 0.053 & 2.46 & 44.3 \\
\hline $\mathrm{H} 55 \beta \ldots \ldots .$. & 74.940 & 0.013 & 0.46 & 52.9 \\
\hline $\mathrm{H} 54 \beta \ldots \ldots .$. & 79.104 & 0.025 & 0.68 & 35.7 \\
\hline $\mathrm{H} 53 \beta \ldots \ldots .$. & 83.582 & 0.011 & 0.41 & 48.5 \\
\hline $\mathrm{H} 52 \beta \ldots \ldots .$. & 88.406 & 0.016 & 0.60 & 60.1 \\
\hline $\mathrm{H} 51 \beta \ldots \ldots .$. & 93.607 & 0.010 & 0.40 & 64.5 \\
\hline $\mathrm{H} 50 \beta \ldots \ldots .$. & 99.225 & 0.013 & 0.53 & 49.8 \\
\hline $\mathrm{H} 49 \beta \ldots \ldots .$. & 105.302 & 0.012 & 0.57 & 65.5 \\
\hline Н37 $\beta \ldots \ldots .$. & 240.021 & 0.013 & 0.54 & 57.5 \\
\hline H36 $\beta \ldots \ldots . .$. & 260.033 & 0.011 & 0.42 & 92.1: \\
\hline $\mathrm{H} 61 \gamma \ldots \ldots \ldots$ & 80.900 & 0.012 & 0.48 & 50.6 \\
\hline $\mathrm{H} 60 \gamma \ldots \ldots .$. & 84.914 & 0.024 & 0.29 : & 17.8: \\
\hline $\mathrm{H} 58 \gamma \ldots \ldots . .$. & 93.776 & 0.009 & 0.28 & 64.1 \\
\hline $\mathrm{H} 57 \gamma \ldots \ldots .$. & 98.671 & 0.006 & 0.16 : & 31.0: \\
\hline $\mathrm{H} 56 \gamma \ldots \ldots .$. & 103.915 & 0.009 & 0.22 : & 35.5: \\
\hline $\mathrm{H} 55 \gamma \ldots \ldots \ldots$ & 109.536 & 0.008 & 0.25 & 35.1 \\
\hline $\mathrm{He} 44 \alpha \ldots \ldots$ & 74.674 & 0.009 & 0.21 & 40.2 \\
\hline $\mathrm{He} 43 \alpha \ldots \ldots$ & 79.945 & 0.012 & 0.17 : & 33.3: \\
\hline $\mathrm{He} 42 \alpha \ldots \ldots$ & 85.723 & 0.023 & 0.37: & 36.3: \\
\hline $\mathrm{He} 41 \alpha \ldots \ldots$ & 92.072 & 0.012 & 0.28 & 27.0 \\
\hline $\mathrm{He} 40 \alpha \ldots \ldots$ & 99.063 & 0.008 & 0.22 & 55.5: \\
\hline $\operatorname{He} 39 \alpha \ldots \ldots$ & 106.781 & 0.005 & 0.18 : & 31.6: \\
\hline $\operatorname{He} 30 \alpha \ldots \ldots$ & 231.995 & 0.009 & 0.23 & 42.0 \\
\hline $\mathrm{He} 29 \alpha \ldots \ldots$ & 256.406 & 0.014 & 0.35 & 35.2 \\
\hline
\end{tabular}

at $18.3 \mathrm{GHz} \mathrm{C}_{3} \mathrm{H}_{2}\left(2_{20}-2_{11}\right)$ at $21.6 \mathrm{GHz}$ transitions, Cox et al. (1987) found $N\left(\mathrm{C}_{3} \mathrm{H}_{2}\right) \sim 3 \times 10^{12} \mathrm{~cm}^{-2}$, which is lower than our value $\left(1.11 \times 10^{14} \mathrm{~cm}^{-2}\right)$ by a factor of about 36 . Given large measurement errors, our value may be unreliable. For $\mathrm{HCS}^{+}$, we obtain a value of $N=4.96 \times 10^{12} \mathrm{~cm}^{-2}$, which should be treated with some caution as it was derived from an extremely faint emission line. From the $\mathrm{N}_{2} \mathrm{H}^{+}(1-0)$ line at $93.2 \mathrm{GHz}$, we derive $N\left(\mathrm{~N}_{2} \mathrm{H}^{+}\right)=2.43 \times 10^{13} \mathrm{~cm}^{-2}$. The column densities of $\mathrm{HCS}^{+}$ and $\mathrm{N}_{2} \mathrm{H}^{+}$have not been reported previously. Our determination of $N\left(\mathrm{HCO}^{+}\right)$is higher than that of Hasegawa \& Kwok (2001) by a factor of 5. Considering the uncertainties in calibrations, such a difference is not unreasonable.

The strong $\mathrm{HCN}$ and $\mathrm{CO}$ transitions are likely to be optically thick and therefore the rotation diagram analysis is not applicable. For these lines we used the following equations to deduce their excitation temperatures and column densities.

The equation of radiative transfer is

$$
T_{s}=\left[I\left(T_{\mathrm{ex}}\right)-I\left(T_{\mathrm{bg}}\right)\right]\left(1-e^{-\tau}\right),
$$

where $I(T)=h \nu\left[k\left(e^{h \nu / k T}-1\right)\right]^{-1}$ with $h$ the Planck constant and $k$ the Boltzmann constant; $\tau$ is the optical depth at the center of a line, and $T_{\mathrm{bg}}$ is the cosmic background temperature $(2.7 \mathrm{~K})$.

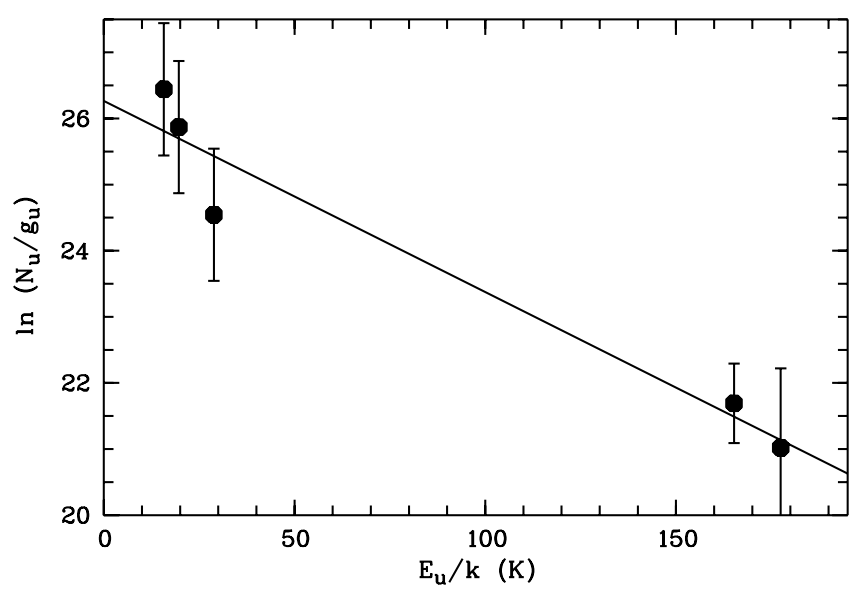

FIG. 7.- Rotational diagram for $\mathrm{HC}_{3} \mathrm{~N}$ in $\mathrm{NGC}$ 7027. The solid line is obtained through a linear least-squares fit of all the data. 
TABLE 5

Excitation Temperatures, Column Densities, and Abundances Relative to $\mathrm{H}_{2}$

\begin{tabular}{|c|c|c|c|c|c|c|}
\hline \multirow[b]{2}{*}{ SPECIES } & \multirow{2}{*}{$\begin{array}{c}T_{\mathrm{ex}} \\
(\mathrm{K})\end{array}$} & \multicolumn{2}{|c|}{$N\left(\mathrm{~cm}^{-2}\right)$} & \multirow[b]{2}{*}{$N($ obs. $) / N($ model $)$} & \multirow[b]{2}{*}{$f_{\mathrm{X}}^{\mathrm{b}}$} & \multirow{2}{*}{$\begin{array}{c}N / f_{\mathrm{X}} \\
\left(\mathrm{cm}^{-2}\right)\end{array}$} \\
\hline & & Obs. & Model $^{\mathrm{a}}$ & & & \\
\hline $\mathrm{HC}_{3} \mathrm{~N}$ & 34.6 & $4.39 \mathrm{E} 13$ & & & $8.5 \mathrm{E}-9$ & $5.2 \mathrm{E} 21$ \\
\hline $\mathrm{CN}$ & & $1.02 \mathrm{E} 14$ & $2.4 \mathrm{E} 14$ & 0.43 & $7.0 \mathrm{E}-8$ & $1.5 \mathrm{E} 21$ \\
\hline $\mathrm{C}_{2} \mathrm{H}$ & & $3.49 \mathrm{E} 14$ & $1.5 \mathrm{E} 14$ & 2.33 & $5.4 \mathrm{E}-8$ & $6.5 \mathrm{E} 21$ \\
\hline $\mathrm{C}_{3} \mathrm{H}_{2} \ldots \ldots \ldots$ & & 1.11E14: & $\ldots$ & $\ldots$ & 8.3E-9: & $1.3 \mathrm{E} 22$ \\
\hline 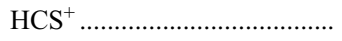 & & $4.96 \mathrm{E} 12$ & $9.0 \mathrm{E} 11$ & 5.51 & $1.0 \mathrm{E}-9$ & $5.0 \mathrm{E} 21$ \\
\hline $\mathrm{N}_{2} \mathrm{H}^{+} \ldots \ldots \ldots \ldots \ldots \ldots \ldots \ldots$ & & $2.43 \mathrm{E} 13$ & $3.2 \mathrm{E} 5$ & $7.59 \mathrm{E} 7$ & $3.8 \mathrm{E}-9$ & $6.4 \mathrm{E} 21$ \\
\hline $\mathrm{HCO}^{+} \ldots \ldots \ldots \ldots \ldots \ldots \ldots \ldots \ldots$ & & $3.38 \mathrm{E} 14$ & $4.6 \mathrm{E} 13$ & 7.35 & $4.8 \mathrm{E}-8$ & $7.0 \mathrm{E} 21$ \\
\hline $\mathrm{H}^{13} \mathrm{CO}^{+} \ldots \ldots \ldots \ldots \ldots \ldots$ & & $7.23 \mathrm{E} 11$ & $\ldots$ & $\ldots$ & $4.1 \mathrm{E}-10$ & $1.8 \mathrm{E} 22$ \\
\hline $\mathrm{HC}^{17} \mathrm{O}^{+} \ldots \ldots \ldots \ldots \ldots \ldots \ldots \ldots$ & & 2.85E13: & $\ldots$ & $\ldots$ & $2.3 \mathrm{E}-9:$ & $1.2 \mathrm{E} 22$ \\
\hline $\mathrm{HC}^{18} \mathrm{O}^{+} \ldots \ldots \ldots \ldots \ldots \ldots \ldots \ldots$ & & 3.89E13: & $\ldots$ & $\ldots$ & $3.2 \mathrm{E}-9$ : & $1.2 \mathrm{E} 22$ \\
\hline $\mathrm{HCN}$ & 5.4 & $7.05 \mathrm{E} 14$ & $1.8 \mathrm{E} 14$ & 3.92 & $4.5 \mathrm{E}-8$ & $1.6 \mathrm{E} 22$ \\
\hline $\mathrm{H}^{13} \mathrm{CN}$ & & $3.65 \mathrm{E} 13$ & $\ldots$ & ... & $3.9 \mathrm{E}-9$ & $9.4 \mathrm{E} 21$ \\
\hline $\mathrm{CO}$ & 40.1 & $3.79 \mathrm{E} 18$ & $3.2 \mathrm{E} 20$ & 0.01 & $1.1 \mathrm{E}-4$ & $3.4 \mathrm{E} 22$ \\
\hline${ }^{13} \mathrm{CO}$ & & $5.19 \mathrm{E} 16$ & $\ldots$ & $\ldots$ & $7.4 \mathrm{E}-6$ & $7.0 \mathrm{E} 21$ \\
\hline $\mathrm{C}^{18} \mathrm{O}$ & & $2.15 \mathrm{E} 15$ & $\ldots$ & $\ldots$ & $3.8 \mathrm{E}-7$ & $5.7 \mathrm{E} 21$ \\
\hline $\mathrm{C}^{17} \mathrm{O}$ & & $5.43 \mathrm{E} 15$ & $\ldots$ & $\ldots$ & $7.4 \mathrm{E}-7$ & 7.3E21 \\
\hline
\end{tabular}

${ }^{\text {a }}$ Calculated by $\int n(i) 4 \pi r^{2} d r / \Delta S$ from Hasegawa et al. (2000), where $\Delta S$ is the projected source area.

${ }^{\mathrm{b}}$ For the species with optically thick emission, this is a lower limit (see text for details).

For the optically thick lines $(\tau \gg 1), T_{\mathrm{ex}}$ can be obtained from equation (2) and is given by

$$
T_{\text {ex }}=\frac{h \nu}{k \ln \left\{h \nu / k\left[T_{s}+I\left(T_{\mathrm{bg}}\right)\right]+1\right\}} .
$$

Assuming that the isotopomer has the same excitation temperature as the main line, we can obtain its optical depth at the line center from equation (2):

$$
\tau_{i}=\ln \left[1-\frac{T_{s}}{I\left(T_{\mathrm{ex}}\right)-I\left(T_{\mathrm{bg}}\right)}\right] .
$$

The column density of the isotopomer can be derived from

$$
N_{i}=\frac{3 h \Delta v \tau_{i} Q\left(T_{\mathrm{ex}}\right) \exp \left(E_{u} / k T_{\mathrm{ex}}\right)}{8 \pi^{3} \mu^{2} S\left[\exp \left(h \nu / k T_{\mathrm{ex}}\right)-1\right]},
$$

where $\Delta v$ is the line width.

Assuming that lines of the isotopomer is optically thin, the $T_{s}$ ratio of the main line and the isotopic line can be derived using equation (3):

$$
\frac{T_{s, m}}{T_{s, i}} \approx \frac{1-\exp \left(-\tau_{m}\right)}{\tau_{i}} .
$$

Once $\tau_{i}$ is obtained from equation (4), the optical depth of the main line, $\tau_{m}$, can be obtained from the above equation.

Finally, we can determine the column density of the main species using

$$
\frac{N_{m}}{N_{i}} \approx \frac{\tau_{m}}{\tau_{i}}
$$

Applying the above equations to $\mathrm{HCN}$ and $\mathrm{CO}$, we obtained their excitation temperatures and column densities. The results are summarized in Table 5. Our determination of the column density of $\mathrm{HCN}$ is a factor of 16 higher than that derived by Hasegawa \& Kwok (2001). In our calculations, a large error may be caused by the very faint $\mathrm{H}^{13} \mathrm{CN}$ line. If applying equation (1), we derive a lower limit of $N(\mathrm{HCN})=4.2 \times 10^{13} \mathrm{~cm}^{-2}$, in agreement with the result of Hasegawa \& Kwok (2001).

For comparison, Table 5 also lists the column densities predicted by the model of Hasegawa et al. (2000). The column densities deduced by current work are in of the same approximate orders of magnitude with the model predictions except for $\mathrm{CO}$ and $\mathrm{N}_{2} \mathrm{H}^{+}$. The model overestimation of the column density of $\mathrm{CO}$ is addressed by Hasegawa et al. (2000) and an improved model (Hasegawa \& Kwok 2001) gives a better fit to the observed column density of $\mathrm{CO}$. The model, however, fails to explain the extremely high column density of $\mathrm{N}_{2} \mathrm{H}^{+}$. A detailed discussion will be presented in the next section.

Assuming a line width of $30 \mathrm{~km} \mathrm{~s}^{-1}$, we estimate the upper limits of the column densities for several nondetected molecular species, and these values are presented in Table 3. Since these upper limits may still serve as useful constraints of the chemical processes in NGC 7027, these upper limits are compared with the model predictions of Hasegawa et al. (2000). Table 3 shows that the observed upper limits are in reasonable agreement with the model predictions except for CS, whose observed column density is lower than the model prediction. Even in the improved model of Hasegawa \& Kwok (2001) the theoretical column density of $N(\mathrm{CS})=8.4 \times 12 \mathrm{~cm}^{-2}$ is still higher than the upper limit derived in the present study.

\subsection{Abundance Calculations}

In order to calculate the fractional abundance of a molecular species relative to $\mathrm{H}_{2}$, we need to know the number density of the species and that of $\mathrm{H}_{2}$, which can be derived by solving the coupled radiative transfer and statistical equilibrium equations. A full treatment of the line excitation requires knowledge of the geometrical structure of the envelope, the rates for collisional excitation of the molecules, and the effects of dust emission and absorption, which however is poorly known, and is beyond the scope of this paper. Instead, assuming that all molecular emission 
originates in a spherical shell, we calculated the molecular abundances respect to $\mathrm{H}_{2}$ using the expression suggested by Olofsson (1996)

$$
f_{\mathrm{X}}=1.7 \times 10^{-28} \frac{v_{e} \theta_{b} D}{\dot{M}_{\mathrm{H}_{2}}} \frac{Q\left(T_{\mathrm{ex}}\right) \nu_{u l}^{2}}{g_{u} A_{u l}} \frac{e^{E_{l} / k T_{\mathrm{ex}}} \int T_{R} d v}{\int_{x_{i}}^{x_{e}} e^{-4 \ln 2 x^{2}} d x},
$$

where the integrated intensity $\int T_{R} d v$ is given in $\mathrm{K} \mathrm{km} \mathrm{s}^{-1}$, the full half power beam width $\theta_{b}$ is in arcseconds, $v_{e}$ is the expansion velocity given in $\mathrm{km} \mathrm{s}^{-1}, D$ is the distance in pc, $\dot{M}_{\mathrm{H}_{2}}$ is the mass loss rate in $M_{\odot} \mathrm{yr}^{-1}, \nu_{u l}$ the line frequency in $\mathrm{GHz}, g_{u}$ the statistical weight of the upper level, $A_{u l}$ the Einstein coefficient for the transition, $E_{l}$ the energy of the lower level, and $x_{i, e}=R_{i, e} /\left(\theta_{b} D\right)$ with $R_{i}$ and $R_{e}$ the inner radius and outer radius of the shell. The equation is deduced under the assumption that the lines are optically thin, $T_{\mathrm{ex}}$ is uniform throughout the shell, the molecular density follows an $r^{-2}$ law, and the shell is formed by a constant mass loss rate and has a constant expansion velocity. One should bear in mind that the spatial variations in the fractional abundances and excitations may be a large source of error. For the calculations, we adopted the excitation temperatures deduced in $\S 4.1$ (see Table 5) and a distance 880 pc to NGC 7027 (Masson 1989). Following the model of Hasegawa et al. (2000) we assumed $R_{i}=0.017 \mathrm{pc}$ and $R_{e}=0.14 \mathrm{pc}$. The expansion velocity was determined from the widths of a few strong lines, which suggest $v_{e} \sim 30 \mathrm{~km} \mathrm{~s}^{-1}$.

Assuming that the $\mathrm{CO}(2-1)$ emission arises from the stellarwind region, we can estimate the mass loss rate from the line strength using the formula given by Winters et al. (2002),

$$
\dot{M}=5.7 \times 10^{-20} \frac{T_{R} v_{e}^{2} D^{2} \theta_{b}^{2}}{S(J) f_{\mathrm{CO}}^{0.85}} M_{\odot} \mathrm{yr}^{-1},
$$

where the correction factor $S(J)=0.5$ for the $\mathrm{CO}(2-1)$ line; the distance $D=880 \mathrm{pc}$; the abundance of CO relative to $\mathrm{H}_{2}$, $f_{\mathrm{CO}}$, is assumed to be $1 \times 10^{-3}$; and the expansion velocity $v_{e}=$ $30 \mathrm{~km} \mathrm{~s}^{-1}$. The derived mass loss rate of $1.1 \times 10^{-4} M_{\odot} \mathrm{yr}^{-1}$ is in good accord with the value of $1.4 \times 10^{-4} M_{\odot} \mathrm{yr}^{-1}$ derived from the model calculations by Sopka et al. (1989). The error in the determination of $\dot{M}$ due to the distance uncertainty is $34 \%$.

Using equation (8), we derived $f_{\mathrm{X}}$ for all the observed molecular species and the results are given in Table 5. The calculated abundance ratios between different species are not rigorously equal to the ratios of column densities. This is due to two reasons. One is the different assumptions made to deconvolve the brightness temperature distribution of the source from the antenna beam. For the calculations of column densities, we have simply assumed that the source brightness distribution is Gaussian. Second, for the abundance calculations, we have assumed that the lines are optically thin. Consequently, when the emission is optically thick, the $f_{\mathrm{X}}$ value given in the table should be treated as a lower limit. We can use the $N(\mathrm{X}) / f_{\mathrm{X}}$ ratio, which approximately gives the column density of $\mathrm{H}_{2}$, to estimate the error due to these assumptions. The last column of Table 5 gives the $N(\mathrm{X}) / f_{\mathrm{X}}$ ratio deduced from each molecular species. These values are generally in good agreement except that derived from $\mathrm{CO}$, which is outside the $3 \sigma$ range. The high $N(\mathrm{CO}) / f_{\mathrm{CO}}$ ratio gives an upper limit of $N\left(\mathrm{H}_{2}\right)$ since the $\mathrm{CO}$ emission is certainly optically thick. Excluding the value deduced from $\mathrm{CO}$, we obtained the average $\mathrm{H}_{2}$ column density of $N\left(\mathrm{H}_{2}\right)=(7.7 \pm 3.8) \times 10^{21} \mathrm{~cm}^{-2}$. Hence, the error due to the assumptions of structure and optically thin emission should be less than a factor of 2. Combining with the uncertainties caused by various parameters (excitation temperature, distance, source size, etc.) and our measurements, we crudely estimate that the total error in the calculations of column density and abundance is a factor of $\sim 8$. The column density ratios and the abundance ratios are expected to be more reliable.

\section{RESULTS AND DISCUSSION}

\subsection{Chemistry}

\subsection{1. $\mathrm{CN}$ and $\mathrm{HCN}$}

Given their relatively large dipole moments, the rotational transitions from the molecules $\mathrm{CN}$ and $\mathrm{HCN}$ are often detected in molecular clouds. HCN can be formed through the dissociative recombination of $\mathrm{HCNH}^{+}$, while $\mathrm{CN}$ is dominantly produced via the photodissociation of $\mathrm{HCN}$ by UV radiation. Therefore, the $\mathrm{CN}$ abundance dramatically increases with increasing stellar radiation when a star evolves beyond the AGB, through the proto-PN stage and to the PN stage. Such a trend has been revealed by a sample study of Bachiller et al. (1997). There is observational evidence showing that the abundance of HCN increases in the PPN stage, and then dramatically decreases in the PN stage (Herpin et al. 2002). This can be attributed to different roles that dissociative recombination and photodissociation play in the two stages. Our observations yield $\mathrm{HCN} /{ }^{13} \mathrm{CO}$ abundance ratios of $>0.008$, consistent the value of $>0.005$ reported by Bachiller et al. (1997). However, we do not find large CN enhancement in NGC 7027. The $\mathrm{CN} / \mathrm{H}_{2}$ abundance ratio is $7.0 \times 10^{-8}$, about a factor of 10 lower than those in AGB stars reported by Woods et al. (2003). The model of Hasegawa et al. (2000) seems to overestimate the $\mathrm{CN}$ abundance by a factor of $2-3$, although this may be explained with the uncertainties in the measurements and calculations. The unexpectedly low $\mathrm{CN}$ abundance was also found by Ali et al. (2001) in their modeling of general PNs.

It has been generally accepted that the $\mathrm{CN} / \mathrm{HCN}$ abundance ratio can trace the UV radiation field. Bachiller et al. (1997) suggest that after a star evolves into the $\mathrm{PN}$ stage, the $\mathrm{CN} / \mathrm{HCN}$ abundance ratio remains constant $(\sim 9)$. We obtain $N(\mathrm{CN}) / N(\mathrm{HCN})<2.4$ in NGC 7027. This probably suggests that the reaction

$$
\mathrm{CN}+\mathrm{H}_{2} \rightarrow \mathrm{HCN}+\mathrm{H}
$$

is very efficient in this young PN.

$$
\text { 5.1.2. } \mathrm{HNC}
$$

$\mathrm{HNC}$ can be formed in a similar way to HCN. Herpin et al. (2002) suggested that in PPN stage the HNC abundance is enhanced with respect to $\mathrm{HCN}$ through ion-molecule reactions. Both observations (Sopka et al. 1989; Bachiller et al. 1997; Josselin \& Bachiller 2003) and models (Ali et al. 2001) indicate that the average abundance ratio $\mathrm{HNC} / \mathrm{HCN}$ in PNs is $\sim 0.5$. However, we do not detect $\mathrm{HNC}$ emission in NGC 7027. The HCN/HNC ratio is estimated to be larger than 30 . The abnormally low $\mathrm{HNC}$ abundance in NGC 7027 was also noted by Bachiller et al. (1997) and Josselin \& Bachiller (2003), who obtained $N(\mathrm{HCN}) / N(\mathrm{HNC})>17$. As proposed by Bachiller et al. (1997), HNC may have been destroyed in NGC 7027 through

$$
\mathrm{HNC}+\mathrm{H} \rightarrow \mathrm{HCN}+\mathrm{H},
$$

which is efficient in high temperatures. The hypothesis is supported by the high $\mathrm{HCN}$ abundance. The model of Hasegawa et al. (2000) did not include the $\mathrm{HNC}$ reaction chains. A more comprehensive model is required to account for the observed $\mathrm{CN} / \mathrm{HCN} /$ HNC abundance ratio. 


\subsection{3. $\mathrm{HC}_{3} \mathrm{~N}$}

$\mathrm{HC}_{3} \mathrm{~N}$ is commonly observed in AGB stars and proto-PNs (e.g., Cernicharo et al. 2000; Pardo et al. 2004). Pardo et al. (2005) found that $\mathrm{HC}_{3} \mathrm{~N}$ is quickly reprocessed from $\mathrm{HCN}$ in PPN stage. Prior to this work, $\mathrm{HC}_{3} \mathrm{~N}$ has never been detected in $\mathrm{PNs}$ and its absence is often explained as the result of efficient photodissociation due to the strong UV radiation in PNs. Thronson \& Bally (1986) estimated the abundance ratio $N\left(\mathrm{HC}_{3} \mathrm{~N}\right) / N(\mathrm{CO}) \leq 1 \times$ $10^{-4}$ in NGC 7027. The abundance upper limit of $\mathrm{HC}_{3} \mathrm{~N}$ is comparable with that in IRC +10216 . They thus did not find evidence for underabundance of $\mathrm{HC}_{3} \mathrm{~N}$ in the $\mathrm{PN}$.

Here we report a possible detection of $\mathrm{HC}_{3} \mathrm{~N}$ in the $\mathrm{PN}$. Our results yield $N\left(\mathrm{HC}_{3} \mathrm{~N}\right) / N(\mathrm{CO})=1.2 \times 10^{-5}$, at lest 1 order of magnitude lower than that in IRC +10216 , confirming the destruction caused by UV photons. The time-dependent chemical model of Ali et al. (2001) predicts that the $\mathrm{HC}_{3} \mathrm{~N}$ abundance gradually decreases with the evolution of PNs. Their model predicted that NGC 6781, a more evolved $\mathrm{PN}$, has a $N\left(\mathrm{HC}_{3} \mathrm{~N}\right) / N(\mathrm{CO})$ ratio of $4.2 \times 10^{-9}$, which is 3 orders of magnitudes lower than that in $\mathrm{NGC} 7027$. The higher abundance of $\mathrm{HC}_{3} \mathrm{~N}$ derived here may be the result of the presence of dense clumps within the nebula, which serve to shield the molecules from the UV radiation from the central star.

\subsection{4. $\mathrm{C}_{2} \mathrm{H}$}

The $\mathrm{C}_{2} \mathrm{H}$ abundance in NGC 7027 is about a factor of 50 lower than that in IRC +10216 . Based on a comparison between the observation and the model prediction of Hasegawa et al. (2000), Hasegawa \& Kwok (2001) concluded that $\mathrm{C}_{2} \mathrm{H}$ is newly produced via the photodissociation of $\mathrm{C}_{2} \mathrm{H}_{2}$ instead of being left over from AGB phase. The $\mathrm{C}_{2} \mathrm{H}$ abundance derived in the present study is in good agreement with the value given by their observation and model.

\subsection{5. $\mathrm{C}_{3} \mathrm{H}_{2}$}

The $\mathrm{C}_{3} \mathrm{H}_{2}$ abundance in NGC 7027 is at least a factor of 4 lower than that in IRC +10216 . Albeit with a low abundance, the large dipole moment makes $\mathrm{C}_{3} \mathrm{H}_{2}$ detectable. Although our detection of $\mathrm{C}_{3} \mathrm{H}_{2}$ is uncertain, the observation of Cox et al. (1987) showed the presence of this species. The abundance of $\mathrm{C}_{3} \mathrm{H}_{2}$ was not predicted by the model of Hasegawa et al. (2000). There is no path for the formation of $\mathrm{C}_{3} \mathrm{H}_{2}$ in $\mathrm{PNs}$, and thus it may be a remnant from $\mathrm{AGB}$ envelope. Based on the observation of molecular clouds, Turner (1991) found that $\mathrm{C}_{3} \mathrm{H}_{2}$ avoids the hot environment and favors the cool clouds. Consequently, to explain its survival from the dissociation of strong UV radiation field in NGC 7027, we infer that $\mathrm{C}_{3} \mathrm{H}_{2}$ may arise from some cool high-density clumps.

$$
\text { 5.1.6. } \mathrm{HCO}^{+} \text {and } \mathrm{N}_{2} \mathrm{H}^{+}
$$

$\mathrm{HCO}^{+}$and $\mathrm{N}_{2} \mathrm{H}^{+}$can be formed through reactions,

$$
\mathrm{H}_{3}^{+}+\mathrm{CO} \rightarrow \mathrm{HCO}^{+}+\mathrm{H}_{2}
$$

and

$$
\mathrm{H}_{3}^{+}+\mathrm{N}_{2} \rightarrow \mathrm{N}_{2} \mathrm{H}^{+}+\mathrm{H}_{2},
$$

which are expected to have a similar rate coefficient. Both $\mathrm{HCO}^{+}$ and $\mathrm{N}_{2} \mathrm{H}^{+}$can be destroyed by dissociative electron recombination. Therefore, if reactions (12) and (13) dominate the production of $\mathrm{HCO}^{+}$and $\mathrm{N}_{2} \mathrm{H}^{+}$, we have the abundance ratio $\mathrm{HCO}^{+} / \mathrm{N}_{2} \mathrm{H}^{+} \approx$
$\mathrm{CO} / \mathrm{N}_{2}$. However, $\mathrm{HCO}^{+}$can be produced through additional processes, such as

$$
\mathrm{CO}^{+}+\mathrm{H}_{2} \rightarrow \mathrm{HCO}^{+}+\mathrm{H}
$$

while $\mathrm{N}_{2} \mathrm{H}^{+}$has no alternative formation channel and can be destroyed at high density by proton transfer reaction with $\mathrm{CO}$,

$$
\mathrm{N}_{2} \mathrm{H}^{+}+\mathrm{CO} \rightarrow \mathrm{HCO}^{+}+\mathrm{N}_{2} .
$$

As a result, the $\mathrm{HCO}^{+} / \mathrm{N}_{2} \mathrm{H}^{+}$ratio is expected to be larger than the $\mathrm{CO} / \mathrm{N}_{2}$ ratio.

The observations of $\mathrm{HCO}^{+}$and $\mathrm{N}_{2} \mathrm{H}^{+}$allow us to test these ion-molecule reactions in NGC 7027. Assuming that the $\mathrm{N}_{2} / \mathrm{H}_{2}$ abundance ratio is equal to $\mathrm{N} / \mathrm{H}\left(1.4 \times 10^{-4}\right.$; Zhang et al. 2005) and $N(\mathrm{CO}) / N\left(\mathrm{H}_{2}\right)=1 \times 10^{-3}$, we have $N(\mathrm{CO}) / N\left(\mathrm{~N}_{2}\right)=7.1$. On the other hand, our observations yield $N\left(\mathrm{HCO}^{+}\right) / N\left(\mathrm{~N}_{2} \mathrm{H}^{+}\right)=14$, higher than the $\mathrm{CO} / \mathrm{N}_{2}$ abundance ratio by a factor of 2 . Hence, additional formation processes for $\mathrm{HCO}^{+}$may be significant.

The $\mathrm{N}_{2} \mathrm{H}^{+}$abundance in NGC 7027 is abnormally high, with an observed column density $7-8$ orders of magnitude higher than predicted by the model of Hasegawa et al. (2000). The species, however, has not been detected in more evolved PNs (Bachiller et al. 1997). Since reaction (13) is the unique path for the production of $\mathrm{N}_{2} \mathrm{H}^{+}$, the high $\mathrm{N}_{2} \mathrm{H}^{+}$abundance therefore implies a high formation rate of $\mathrm{H}_{3}^{+} \cdot \mathrm{H}_{3}^{+}$can be produced through ionization of $\mathrm{H}_{2}$ by cosmic-ray or soft X-ray emission from the central star. Since strong X-ray emission from NGC 7027 has been detected (Kastner et al. 2001), it is possible that X-ray emission may play an key role in the formation of $\mathrm{N}_{2} \mathrm{H}^{+}$in $\mathrm{NGC} 7027$. This is consistent with the fact that more evolved PNs generally have weaker X-ray emission and have no $\mathrm{N}_{2} \mathrm{H}^{+}$emission. If high $\mathrm{N}_{2} \mathrm{H}^{+}$ abundance is a property of young PNs, further observations of $\mathrm{N}_{2} \mathrm{H}^{+}$in a sample of young PNs would be useful.

Although $\mathrm{N}_{2} \mathrm{D}^{+}$has been extensively observed in dark clouds, we are unable to detect it in NGC 7027. From the $3 \sigma$ intensity upper limit of the $\mathrm{N}_{2} \mathrm{D}^{+}(1-0)$ transition, we estimate that NGC 7027 has a fractionation ratio $N\left(\mathrm{~N}_{2} \mathrm{D}^{+}\right) / N\left(\mathrm{~N}_{2} \mathrm{H}^{+}\right)<0.1$. The lower fractionation ratio in NGC 7027 compared to that found in dark clouds (Daniel et al. 2007) can be attributed to different physical environments.

$\mathrm{HCO}^{+}$in NGC 7027 has a similarly high abundance to that in older PNs. The $\mathrm{HCO}^{+} / \mathrm{HCN}$ abundance ratio in NGC 7027 is 0.5 , which is in very good agreement with the average value for older PNs (Bachiller et al. 1997). The different behaviors of $\mathrm{HCO}^{+}$and $\mathrm{N}_{2} \mathrm{H}^{+}$in young and more evolved PNs suggest that reaction (12) is not the dominant process for the formation of $\mathrm{HCO}^{+}$.

\subsection{7. $\mathrm{HCS}^{+}$and $\mathrm{CS}$}

We obtain a $N\left(\mathrm{HCO}^{+}\right) / N\left(\mathrm{HCS}^{+}\right)$column density ratio of 68 , which is comparable to the ion abundance ratio of $N\left(\mathrm{O}^{+}\right) / N\left(\mathrm{~S}^{+}\right)=$ 111 derived in the ionized regions of NGC 7027 (Zhang et al. 2005). Hence, the formation rate of $\mathrm{HCS}^{+}$should be similar to that of $\mathrm{HCO}^{+}$.

The estimated CS abundance is about a factor of 10 lower than the model value of 53 (Hasegawa et al. 2000). According to Herpin et al. (2002), after a star leaves the AGB strong shocks can occur and significantly affect the circumstellar chemistry. Willacy \& Cherchneff (1998) suggest that shocks can destroy $\mathrm{CS}$ and $\mathrm{HCN}$ in circumstellar envelopes, which might imply that the low CS abundance observed in NGC 7027 is due to the presence of shocks. 


\subsection{Isotopic Ratios}

\subsubsection{Carbon}

The determination of isotopic abundances plays an important role in our understanding of nucleosynthesis in evolved stars. Extensive studies have shown that the ${ }^{12} \mathrm{C} /{ }^{13} \mathrm{C}$ abundance ratios in red giant stars are considerably lower than the prediction made by standard stellar evolution models (e.g., Charbonnel \& do Nascimento 1998). An extra mixing mechanism, called cool bottom processing (CBP), was introduced to explain the low ${ }^{12} \mathrm{C} /{ }^{13} \mathrm{C}$ ratio (Sackmann \& Boothroyd 1999; Boothroyd \& Sackmann 1999). For the stars with masses in the range $2.5 \leq M / M_{\odot} \leq 6$, the hot bottom burning (HBB) may occur during the AGB stage and induce ${ }^{12} \mathrm{C} /{ }^{13} \mathrm{C}$ to further decrease to $\sim 3.5$ (Frost et al. 1998). A few studies have been made to study ${ }^{12} \mathrm{C} /{ }^{13} \mathrm{C}$ in molecular envelope of PNs (e.g., Palla et al. 2000; Balser et al. 2002) and lend support to the existence of CBP.

Three ${ }^{13} \mathrm{C}$-bearing molecular species, ${ }^{13} \mathrm{CO}, \mathrm{H}^{13} \mathrm{CN}$, and $\mathrm{H}^{13} \mathrm{CO}^{+}$, have been detected in our observations, allowing us to estimate the ${ }^{12} \mathrm{C} /{ }^{13} \mathrm{C}$ ratio. The integrated intensity ratio of the $\mathrm{CO}(2-1)$ and ${ }^{13} \mathrm{CO}(2-1)$ is 25 , yielding an abundance ratio of $N\left({ }^{12} \mathrm{CO}\right) / N\left({ }^{13} \mathrm{CO}\right)=73$. Varying values of the ${ }^{12} \mathrm{CO} /{ }^{13} \mathrm{CO}$ ratio in NGC 7027 have been reported in the literature. Knapp \& Chang (1985) obtained $40 \lesssim N\left({ }^{12} \mathrm{CO}\right) / N\left({ }^{13} \mathrm{CO}\right) \lesssim 130$. Sopka et al. (1989) did not detect the ${ }^{13} \mathrm{CO}$ emission in NGC 7027 and estimated ${ }^{12} \mathrm{CO} /{ }^{13} \mathrm{CO}$ to be larger than 100 . Kahane et al. (1992), Bachiller et al. (1997), and Josselin \& Bachiller (2003) got a lower limit of 65,25 , and 11 , respectively. More recently, Balser et al. (2002) obtained that $N\left({ }^{12} \mathrm{CO}\right) / N\left({ }^{13} \mathrm{CO}\right) \sim 31$, about half of our determination.

The $\mathrm{HCN} / \mathrm{H}^{13} \mathrm{CN}$ abundance ratio is 19 , similar to the $I[\mathrm{HCN}(3-2)] / I\left[\mathrm{H}^{13} \mathrm{CN}(3-2)\right]$ integrated intensity ratio of 15 . The value may bear a larger error due to the weakness of the $\mathrm{H}^{13} \mathrm{CN}(3-2)$ transition. This is for the first time that the isotopomer ratio of this species in NGC 7027 is obtained. The value is a factor of $\sim 4$ lower than the ${ }^{12} \mathrm{CO} /{ }^{13} \mathrm{CO}$ ratio. The higher ${ }^{12} \mathrm{CO} /{ }^{13} \mathrm{CO}$ ratio suggests that ${ }^{13} \mathrm{CO}$ might be partly affected by selective photodissociation of the UV radiation field, as proposed by Knapp \& Chang (1985). Since both the CO and HCN lines are likely to be optically thick, the above values may represent lower limits. If the ${ }^{12} \mathrm{C} /{ }^{13} \mathrm{C}$ ratio is indeed as low as suggested above, this may imply a nonstandard mixing process such as CBP.

Alternatively, the $\mathrm{HCO}^{+} / \mathrm{H}^{13} \mathrm{CO}^{+}$ratio can be used. The $\mathrm{HCO}^{+}$lines are weaker but are definitely optically thin. We used the $\mathrm{HCO}^{+}(1-0)$ line at $89.188 \mathrm{GHz}$ and the $\mathrm{H}^{13} \mathrm{CO}^{+}(3-2)$ line at $260.255 \mathrm{GHz}$ since the $\mathrm{H}^{13} \mathrm{CO}^{+}(1-0)$ transition at $88.632 \mathrm{GHz}$ falls within a spectral region that has a poor signal-to-noise ratio and the $\mathrm{HCO}^{+}(3-2)$ emission at $267.558 \mathrm{GHz}$ falls out of our frequency ranges. Our calculations yield a rather high $\mathrm{HCO}^{+}$/ $\mathrm{H}^{13} \mathrm{CO}^{+}$abundance ratio of 117 (see Table 5). If the $\mathrm{HCO}^{+}(1-0)$ transition is optically thick, the value is even higher. On the other hand, based on the $3 \sigma$ upper limit of the integrated intensity of the $\mathrm{H}^{13} \mathrm{CO}^{+}(1-0)$ line, we estimate that $N\left(\mathrm{HCO}^{+}\right) / N\left(\mathrm{H}^{13} \mathrm{CO}^{+}\right)>$ 60 . The $\mathrm{HCO}^{+} / \mathrm{H}^{13} \mathrm{CO}^{+}$ratio derived in the present study is higher than the value of 40 given by Hasegawa \& Kwok (2001). The abnormal carbon isotopic ratio derived from $\mathrm{HCO}^{+}$probably suggests that chemical fractionation is significant for this species.

\subsubsection{Oxygen}

During the AGB stage, the nucleosynthesis and dredge-up processes cause destruction of ${ }^{18} \mathrm{O}$ and enhancement of ${ }^{17} \mathrm{O}$ at the surface of evolved stars (see Busso 2006 for a recent review). Knapp \& Chang (1985) detected the oxygen isotopic ratio for a sample of carbon-rich circumstellar envelopes and found the range of $300-800$ and $300-1300$ for ${ }^{16} \mathrm{O} /{ }^{17} \mathrm{O}$ and ${ }^{16} \mathrm{O} /{ }^{18} \mathrm{O}$, respectively. Based on the fact that the ${ }^{17} \mathrm{O} /{ }^{18} \mathrm{O}$ ratios in carbonrich envelopes are higher than that in the interstellar medium by a factor of 4-5, they ruled out AGB stars as the dominant source of oxygen in the interstellar medium.

From the detection of $\mathrm{CO}, \mathrm{C}^{17} \mathrm{O}$, and $\mathrm{C}^{18} \mathrm{O}$ emission in $\mathrm{NGC}$ 7027, we obtain the oxygen isotopic ratios $N\left({ }^{16} \mathrm{O}\right) / N\left({ }^{17} \mathrm{O}\right)=698$, $N\left({ }^{16} \mathrm{O}\right) / N\left({ }^{18} \mathrm{O}\right)=1763$, and $N\left({ }^{17} \mathrm{O}\right) / N\left({ }^{18} \mathrm{O}\right)=3$. Our results are consistent with the ratios (or the lower limits) derived by Knapp \& Chang (1985) in NGC 7027. Compared with the oxygen isotopic ratios in the $\operatorname{Sun}\left(N\left({ }^{16} \mathrm{O}\right) / N\left({ }^{17} \mathrm{O}\right)=0.2, N\left({ }^{16} \mathrm{O}\right) / N\left({ }^{18} \mathrm{O}\right)=499\right.$, and $N\left({ }^{17} \mathrm{O}\right) / N\left({ }^{18} \mathrm{O}\right)=2682$; Lodders 2003$)$, NGC 7027 clearly shows enhancement of ${ }^{17} \mathrm{O}$ and destruction of ${ }^{18} \mathrm{O}$, which well agrees with the predictions of stellar models.

\subsection{Hydrogen and Helium Recombination Lines}

A total of over $30 \mathrm{H}$ and $\mathrm{He}$ recombination lines are detected in our observations. These include nine $\mathrm{H} n \alpha$ lines, nine $\mathrm{H} n \beta$ lines, and probably six $\mathrm{H} n \gamma$ lines and eight $\mathrm{He} \alpha \alpha$ lines. The line widths and integrated intensities of these lines are listed in Table 4.

Due to optical depth effects, the line widths and radial velocities of recombination lines at different frequencies may be different (Ershov \& Berulis 1989). The high-frequency emission has negligible optical depth, and is likely to be emitted from the whole ionized region. The low-frequency emission, however, is more likely to be optically thick and if so will be emitted from the outer regions. Our data do not show any clear correlation between the line frequencies and the line widths and velocities, suggesting that these recombination lines are optically thin. From profile fittings, we determine the averaged line widths (FWHM) for $\mathrm{H} n \alpha$, $\mathrm{H} n \beta$ and Hen $\alpha$ lines to be $44 \pm 8 \mathrm{~km} \mathrm{~s}^{-1}, 54 \pm 9 \mathrm{~km} \mathrm{~s}^{-1}$, and $38 \pm 9 \mathrm{~km} \mathrm{~s}^{-1}$, respectively. No systematic difference is found for the widths of hydrogen lines and the helium lines. The Hno lines detected here are a factor of about 1.6 narrower than the lowfrequency $\mathrm{H} 110 \alpha$ line detected by Chaisson \& Malkan (1976), which is expected to be optically thick. The observed widths of hydrogen radio recombination lines can be interpreted by the model of Ershov \& Berulis (1989; cf. their Fig. 2c).

The relative intensities of observed $\mathrm{H} n \alpha$ lines as a function of their quantum numbers are plotted in Figure 8. In this plot, we have corrected for the effect of beam dilution and translated the intensity unit into Jy. The low-frequency lines $\mathrm{H} 76 \alpha$ and $\mathrm{H} 110 \alpha$ reported by Chaisson \& Malkan (1976) are also plotted in this figure. We have normalized the intensities of the H76 $\alpha$ and H1 $10 \alpha$ lines to $I(\mathrm{H} 39 \alpha)=1$ by assuming that they have a same beam-filling factor, which may introduce an error less than a factor of 5. For comparison, we plot the theoretical predictions of Storey \& Hummer (1995) under the assumption of Case B with several combinations of electron temperatures and densities. Note that Storey \& Hummer (1995) only gives the line emissivities for $n \leq 50$ and the predictions for $n>50$ in Figure 8 are linear extrapolations of their results. If a correction for continuum free-free opacity is applied, the predicted lines would have a larger slope than those shown in Figure 8. An inspection of Figure 8 shows that the intensities of the high-frequency $\mathrm{H} n \alpha$ lines $(>50 \mathrm{GHz})$ are consistent with the theory of recombination and are not affected by free-free opacity. The fact that the high-frequency $\mathrm{H} n \alpha$ lines are weaker than the theoretical predictions may suggest that they are optically thick.

$\mathrm{H} n \alpha$ and $\mathrm{H} n \beta$ radio recombination lines were also detected in molecular clouds (e.g., Turner 1991; Nummelin et al. 2000), allowing us to make a comparison study. The averaged mainbeam temperatures for $\mathrm{H} n \alpha$ lines and $\mathrm{H} n \beta$ lines determined from our observations are $T(\alpha)=0.046 \mathrm{~K}$ and $T(\beta)=0.014 \mathrm{~K}$, 


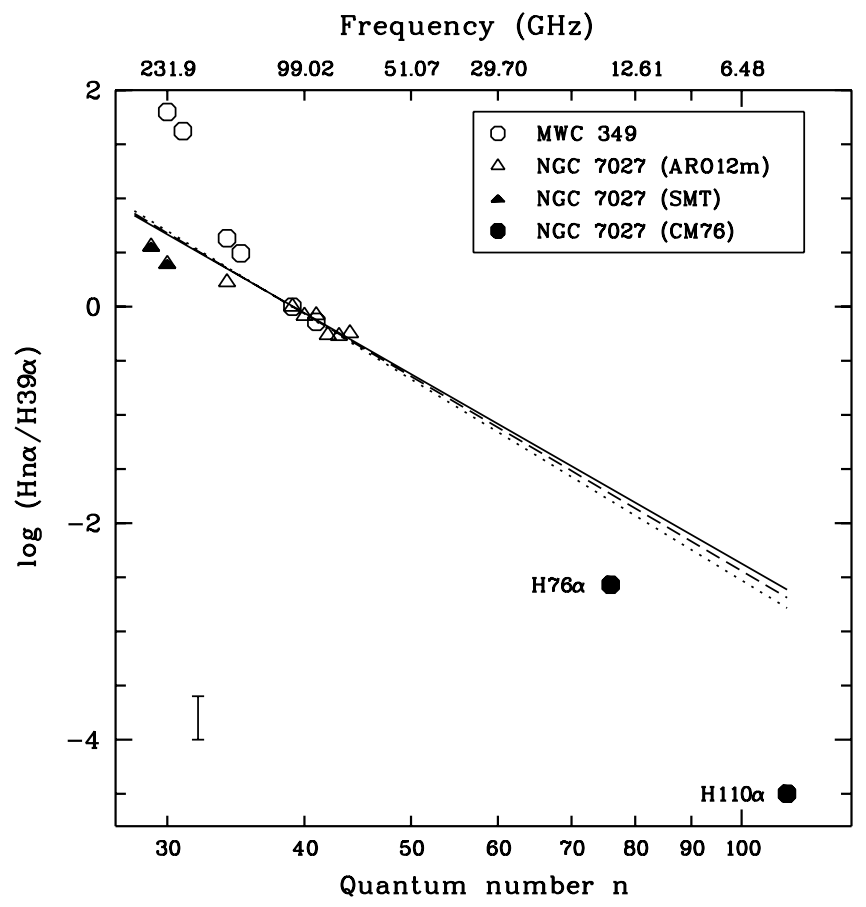

FIG. 8. - Relative fluxes of $\mathrm{H}$ I recombination $\alpha$-transitions in MWC 349 (open circles; Thum et al. 1998) and NGC 7027. The open triangles and the filled triangles are from our observations of NGC 7027 with the ARO $12 \mathrm{~m}$ and the SMT $10 \mathrm{~m}$ telescopes, respectively. The error bar on the lower left indicates typical uncertainties of our observations. The filled circles are from the observations of NGC 7027 by Chaisson \& Malkan (1976). The theoretical predictions of Storey \& Hummer (1995) are given by assuming $T_{e}=12,500 \mathrm{~K}$ and $N_{e}=10^{4} \mathrm{~cm}^{-3}$ (solid line), $T_{e}=12,500 \mathrm{~K}$ and $N_{e}=10^{3} \mathrm{~cm}^{-3}$ (dotted line), and $T_{e}=10,000 \mathrm{~K}$ and $N_{e}=10^{4} \mathrm{~cm}^{-3}$ (dashed line). No correction for free-free continuum opacity is included.

respectively. The corresponding $T_{R}(\alpha) / T_{R}(\beta)$ ratio in NGC 7027 is 3.3, which is in good agreement with that found in two molecular clouds OMC-1 and Sgr B2 (Turner 1991). According to Turner (1991), stimulated emission must be significant in order to explain such a $T_{R}(\alpha) / T_{R}(\beta)$ ratio. The observed $T_{R}(\alpha)$ value in NGC 7027 is lower than that in OMC-1 by a factor of 10, which can be attributed to the smaller size $(\sim 0.1 \mathrm{pc})$ of this PN. Following the calculations of Turner (1991), NGC 7027 has an emission measure $\mathrm{EM} \sim 6 \times 10^{6} \mathrm{~cm}^{-6} \mathrm{pc}$.

Thum et al. (1998) studied the radio recombination lines of hydrogen in the emission-line star MWC 349 and found that the amplification factor of the recombination line maser has a peak value near $n=19$. Figure 8 compares hydrogen recombination lines in MWC 349 and those in NGC 7027. We can clearly see that for MWC 349, the intensities of the Hn $\alpha$ lines near $n=30$ are consistently higher than the theoretical predictions, which has been attributed to the broadband maser phenomenon (Thum et al. 1998). The trend is not found in NGC 7027. From the $T(\alpha) / T(\beta)$ ratio, we find stimulated emission to be significant for the hydrogen radio recombination in NGC 7027 and infer that all the $\mathrm{H} n \alpha$ lines observed in the present work have a similar amplification factor. NGC 7027 has a density of $10^{4} \mathrm{~cm}^{-3}$, about a factor of $10^{4}$ lower than that of MWC 349. Consequently, according to the model of Thum et al. (1998), the quantum number of the peak amplification $\left(n_{\max }\right)$ in NGC 7027 is larger than that in MWC 349. Since $n_{\max }=19 \pm 2$ in MWC 349, a similar amplification factor for the Hno would imply that $n_{\max }$ is in the range between 34 and 45 in NGC 7027.

The hydrogen and helium radio recombination lines can also be used to calculate the $\mathrm{He}^{+} / \mathrm{H}^{+}$abundance ratio. Palmer et al.
(1969) deduced the $\mathrm{He} / \mathrm{H}$ abundance ratio from radio recombination for a sample of $\mathrm{H}$ II regions and found that the results are in good agreement with those derived from optical data. Following Palmer et al. (1969) and assuming that the ratio of the integrated intensity of the Hen $\alpha$ line to that of the $\mathrm{H} n \alpha$ represents the $\mathrm{He}^{+} / \mathrm{H}^{+}$abundance ratio, we obtain $N\left(\mathrm{He}^{+}\right) / N\left(\mathrm{H}^{+}\right)=0.11 \pm$ 0.02 . An additional $20 \%$ error is given by uncertainties in the measurements. The derived $\mathrm{He}^{+} / \mathrm{H}^{+}$abundance ratio is about a factor of 2 higher than that determined from the optical spectra (Zhang et al. 2005). A possible explanation is that the $\mathrm{He}^{+}$zone is more extended than the $\mathrm{H}^{+}$zone so that the effect of beam dilution is more severe for the $\mathrm{H}$ I radio recombination lines. To account for the discrepancy between the abundance derived from the radio data and that from the optical data, the size of the $\mathrm{He}^{+}$ zone may be larger than the $\mathrm{H}^{+}$zone by a factor of about 1.3. This is possible if the ionization spectrum is hard enough and more UV photons have an energy close to the ionization potential of helium. In that case, the $\mathrm{He}^{2+}$ zone is expected to be large in the high-excitation PNs. However, the He II radio recombination lines are below our detection limit, and thus we have not attempted to calculate the elemental abundance ratio of helium to hydrogen.

\subsection{Line Profiles}

Figures 5 and 6 present the line profiles detected with the ARO $12 \mathrm{~m}$ telescope and the SMT $10 \mathrm{~m}$ telescope, respectively. The $\mathrm{HCO}^{+}$line exhibits a self-absorption feature at $V_{\mathrm{LSR}} \sim 22 \mathrm{~km} \mathrm{~s}^{-1}$ in accord with the observations of $\mathrm{HCO}^{+}$line by Deguchi et al. (1990). We also find a weak absorption for the HCN lines at $V_{\mathrm{LSR}} \sim 30 \mathrm{~km} \mathrm{~s}^{-1}$.

Table 1 gives the FWHM of detected molecular lines. The emission lines from $\mathrm{CO}$ and its isotopomers have a width between 23 and $30 \mathrm{~km} \mathrm{~s}^{-1}$. Hasegawa \& Kwok (2001) found that the $\mathrm{HCN}, \mathrm{CN}$, and $\mathrm{C}_{2} \mathrm{H}$ have larger line widths than that of the $\mathrm{CO}$ emission, even after taking into account the effects of hyperfine components. Their findings are confirmed by our observations.

One possible explanation for the larger line widths is that these molecular lines contain components arising from fast-moving clumps within the neutral envelope of NGC 7027 (Redman et al. 2003; Huggins \& Mauron 2002). The CO emission originates in a much more extended region compared to other molecular species, and thus is less affected by the contribution from the fast components. The present observations show that the lines from $\mathrm{HCO}^{+}$and $\mathrm{N}_{2} \mathrm{H}^{+}$are consistently narrower than those from $\mathrm{CO}$. We therefore infer that the fast clumps might be neutral and have no contribution to the emission from the molecular ions. The molecular ions mostly originate from the PDR, which has a lower expansion velocity. Further observations of lines from molecular ions are required to verify this point. Figures 5 and 6 show that the recombination lines are generally broader than $\mathrm{CO}$ lines, suggesting that the acceleration by the UV radiation field is significant in the ionized regions of NGC 7027.

\subsection{Comparison with the Spectra of IRC+10216 and CRL 618}

Comparison with the spectra of AGB stars, PPNs, and PNs can provide essential information of chemical evolution in latetype stars. Recent molecular line surveys of IRC+10216 and CRL 618 were presented by Cernicharo et al. (2000) and Pardo et al. (2007), respectively. The frequency ranges are $129-172.5 \mathrm{GHz}$ for IRC+10216, and 80.25-115.75, 131.25-179.25, and 204.25275.25 GHz for CRL 618. Since these spectra were obtained using different instruments, it is hard to make a quantitative comparison with our observation of NGC 7027. Through a qualitative 
comparison, we summarize the main differences between the spectra of the three objects as follows.

1. The molecular lines detected in NGC 7027 are much fewer and generally fainter than those in IRC+10216 and CRL 618, even after the correction for the relative distances of the three objects. This can be attributed to (1) the low density in the neutral envelope of NGC 7027, which makes it harder to collisionally excite the higher rotational levels of most molecules (Thronson \& Bally 1986), and (2) destruction of molecules caused by the strong UV radiation and shock waves in NGC 7027.

2. Refractory metal-bearing and silicon-bearing species which are plentifully present in IRC+10216 have only weak emission in CRL 618 and are not detected in NGC 7027. These molecules may be depleted onto dust grains with stellar evolution.

3. The CS emission is strong in IRC +10216 , medium in CRL 618 , and not seen in NGC 7027. This might suggest that shock waves play an important role in the chemistry of NGC 7027.

4. Ionized species $\left(\mathrm{HCO}^{+}, \mathrm{HCS}^{+}\right.$, and $\left.\mathrm{N}_{2} \mathrm{H}^{+}\right)$and recombination lines detected in CRL 618 and NGC 7027 are not seen in the spectra of IRC +10216 . This is due to photoionization by the hotter central stars of CRL 618 and NGC 7027.

5. The $\mathrm{N}_{2} \mathrm{H}^{+} / \mathrm{HCO}^{+}$abundance ratio in NGC 7027 is about a factor of 30 higher than that in CRL 618, supporting the hypothesis that $\mathrm{H}_{3}^{+}$is dominantly produced by soft X-ray from the central star.

The comparison between the spectra of CRL 618 and NGC 7027 is particularly meaningful because the former is a much evolved PPN and will evolve to become a young PN like NGC 7027 after only a few hundred years. From the above comparison, we can conclude that the chemical compositions can dramatically change after the PPN stage.

\section{SUMMARY}

In this spectral-line survey of the PN NGC 7027, we have detected a total of 67 spectral lines consisting of 21 molecular lines, 32 hydrogen and helium recombination lines, and 14 unidentified lines. The line intensities were used to calculate excitation temperatures, column densities, and fractional abundances of the observed molecular species. The main findings are summarized as follows.

1. The low $\mathrm{CN} / \mathrm{HCN}$ ratio seems to go against the theoretical expected photodissociation of $\mathrm{HCN}$ into $\mathrm{CN}$ in a strong UV environment. This could imply that the photodissociation process might be compensated by the unexpectedly efficient reaction between $\mathrm{CN}$ and $\mathrm{H}_{2}$. Alternatively, a large amount of $\mathrm{HNC}$ may have been transferred into $\mathrm{HCN}$ in the hot envelope of NGC 7027.

2. From the $\mathrm{HCO}^{+} / \mathrm{N}_{2} \mathrm{H}^{+}$abundance ratio, we suggest that the reaction of $\mathrm{CO}^{+}$and $\mathrm{H}_{2}$ may be important for the production of
$\mathrm{HCO}^{+}$. The soft X-ray emission from the central star play to important role for the production of $\mathrm{N}_{2} \mathrm{H}^{+}$in NGC 7027 .

3. We find evidence for underabundance of CS, suggesting that the species may have been destroyed by shocks in the young PN.

4. Although the estimated $\mathrm{CO} /{ }^{13} \mathrm{CO}, \mathrm{HCN} / \mathrm{H}^{13} \mathrm{CN}$, and $\mathrm{HCO}^{+} /$ ${ }^{13} \mathrm{CO}^{+}$ratios are different, the resulting low ${ }^{12} \mathrm{C} /{ }^{13} \mathrm{C}$ ratio supports the presence of extra mixing process in $\mathrm{AGB}$ stage. The oxygen isotopomer ratios is consistent with the predictions of stellar models.

5. The intensities of hydrogen recombination lines suggest that stimulated excitation may be significant. Our estimates of $\mathrm{He}^{+} / \mathrm{H}^{+}$abundance ratio from the ratio recombination lines is higher than that from the optical lines. The discrepancy can be explained if the $\mathrm{He}^{+}$zone is more extended that the $\mathrm{H}^{+}$zone in the young PN.

6. The different widths of lines from $\mathrm{CO}$, other neutral molecules, molecular ions, and hydrogen and helium can be used as dynamical probes of the circumstellar envelope of NGC 7027. Our results suggest the presence of fast neutral clumps within the molecular envelope of NGC 7027.

These observations show that the circumstellar envelope of NGC 7027 can serve as a very useful chemical laboratory. Comparison with existing theoretical chemical models suggests that a more comprehensive model is required to explain the observed molecular abundances, in particular for the production of $\mathrm{N}_{2} \mathrm{H}^{+}$, the destruction of $\mathrm{CS}$, and the survival of $\mathrm{HC}_{3} \mathrm{~N}$ and $\mathrm{C}_{3} \mathrm{H}_{2}$ in the PN. A more comprehensive model will need to include effects of soft X-ray emission from the central star, shocks, and dense clumps.

Changing physical conditions are the main factor for the different molecular abundances in the circumstellar envelopes of stars at different evolutionary stages. The observational results reported in the present paper provide a foundation for further studies of circumstellar chemistry. In this spectral survey program, we also obtained radio spectra of two AGB stars, IRC+10216 and CIT 6, and one proto-PN, CRL 2688, in addition to the PN NGC 7027. A systematic comparison of the chemical compositions in different evolutionary stages will be presented in a forthcoming paper.

We thank Aldo Apponi for his help in the processing of the ARO data. We also thank Jun-ichi Nakashima and Jin-Hua He for useful discussions. This work is supported in part by a grant awarded to S. K. from the Research Grants Council of Hong Kong. D. V. T. acknowledges support of this work by Academia Sinica, Taiwan, and the Natural Science Council of Taiwan.
Ali, A., Shalabiea, O. M., El-Nawawy, M. S., \& Millar, T. J. 2001, MNRAS, 325,881

Bachiller, R., Forveille, T., Huggins, P. J., \& Cox, P. 1997, A\&A, 324, 1123

Balser, D. S., McMullin, J. P., \& Wilson, T. L. 2002, ApJ, 572, 326

Boothroyd, A. I., \& Sackmann, I.-J. 1999, ApJ, 510, 232

Busso, M. M. 2006, in IAU Symp. 234, Planetary Nebulae, ed. M. J. Barlow \&

R. H. Méndez (Cambridge: Cambridge Univ. Press), 91

Cernicharo, J., Guélin, M., \& Kahane, C. 2000, A\&AS, 142, 181

Cernicharo, J., Heras, A. M., Pardo, J. R., Tielens, A. G. G. M., Guélin, M., Dartois, E., Neri, R., \& Waters, L. B. F. M. 2001a, ApJ, 546, L127

Cernicharo, J., Heras, A. M., Tielens, A. G. G. M., Pardo, J. R., Herpin, F., Guélin, M., \& Waters, L. B. F. M. 2001b, ApJ, 546, L123

Chaisson, E. J., \& Malkan, A. M. 1976, ApJ, 210, 108

Charbonnel, C., \& do Nascimento, J. D. 1998, A\&A, 336, 915

Cox, P., Bachiller, R., Huggins, P. J., Omont, A., \& Guilloteau, S. 1993, in IAU Symp. 155, Planetary Nebulae, ed. R. Weinberger \& A. Acker (Dordrecht: Kluwer), 227
Cox, P., Gusten, R., \& Henkel, C. 1987, A\&A, 181, L19

Daniel, F., Cernicharo, J., Roueff, E., Gerin, M., \& Dubernet, M. L. 2007, ApJ, 667,980

Deguchi, S., Claussen, M. J., \& Goldsmith, P. F. 1986, ApJ, 303, 810

Deguchi, S., Izumiura, H., Kaifu, N., Mao, X., Nguyen-Q-Rieu, \& Ukita, N. 1990, ApJ, 351, 522

Ershov, A. A., \& Berulis, I. I. 1989, Pis'ma Astron. Zh., 15, 413

Frost, C. A., Cannon, R. C., Lattanzio, J. C., Wood, P. R., \& Forestini, M. 1998, A\&A, 332, L17

Hasegawa, T. I., \& Kwok, S. 2001, ApJ, 562, 824

Hasegawa, T. I., Volk, K., \& Kwok, S. 2000, ApJ, 532, 994

Herpin, F., Goicoechea, J. R., \& Cernicharo, J. 2002, ApJ, 577, 961

Huggins, P. J., \& Mauron, N. 2002, A\&A, 393, 273

Josselin, E., \& Bachiller, R. 2003, A\&A, 397, 659

Kahane, C., Cernicharo, J., Gómez-González, J., \& Guélin, M. 1992, A\&A, 256,235 
Kastner, J. H., Vrtilek, S. D., \& Soker, N. 2001, ApJ, 550, L189

Knapp, G. R., \& Chang, K. M. 1985, ApJ, 293, 281

Kwok, S. 2004, Nature, 430, 985

Lilley, A, E., \& Palmer, P. 1968, ApJS, 16, 143

Liu, X.-W., et al. 1996, A\&A, 315, L257

Lodders, K. 2003, ApJ, 591, 1220

Masson, C. R. 1989, ApJ, 336, 294

Masson, C. R., et al. 1985, ApJ, 292, 464

Müller, H. S. P., Schlöder, F., Stutzki, J., \& Winnewisser, G. 2005, J. Mol. Struct., 742, 215

Müller, H. S. P., Thorwirth, S., Roth, D. A., \& Winnewisser, G. 2001, A\&A 370, L49

Nummelin, A., et al. 1998, ApJS, 117, 427 2000, ApJS, 128, 213

Olofsson, H. 1996, in IAU Symp. 178, Molecules in Astrophysics: Probes \& Processes, ed. E. van Dishoeck (Dordrecht: Kluwer), 457 1997, Ap\&SS, 251, 31

Palla, F., Bachiller, R., Stanghellini, L., Tosi, M., \& Galli, D. 2000, A\&A, 355, 69

Palmer, P., Zuckerman, B., Penfield, H., \& Lilley, A. E. 1969, ApJ, 156, 887

Pardo, J. R., \& Cernicharo, J. 2007, ApJ, 654, 978

Pardo, J. R., Cernicharo, J., \& Goicoechea, J. R. 2005, ApJ, 628, 275

Pardo, J. R., Cernicharo, J., Goicoechea, J. R., Gu'elin, M., \& Ramos, A. A. 2007, ApJ, 661, 250

Pardo, J. R., Cernicharo, J., Goicoechea, J. R., \& Phillips, T. G. 2004, ApJ, 615, 495
Pickett, H. M., Poynter, R. L., Cohen, E. A., Delitsky, M. L., Pearson, J. C., \& Muller, H. S. P. 1998, J. Quant. Spectrosc. Radiat. Transfer, 60, 883

Redman, M. P., Viti, S., Cau, P., \& Williams, D. A. 2003, MNRAS, 345, 1291

Roelfsema, P. R., Goss, W. M., Zijlstra, A., \& Pottasch, S. R. 1991, A\&A, 251, 611

Sackmann, I.-J., \& Boothroyd, A. I. 1999, ApJ, 510, 217

Sopka, R. J., Olofsson, H., Johansson, L. E. B., Nguyen-Q-Rieu, \& Zuckerman, B. 1989, A\&A, 210, 78

Storey, P. J., \& Hummer, D. 1995, MNRAS, 272, 41

Thronson, H. A., \& Bally, J. 1986, ApJ, 300, 749

Thum, C., Marín-Pintado, J. Quirrenbach, A., \& Matthews, H. E. 1998, A\&A, 333, L63

Towle, J. P., Feldman, P. A., \& Watson, J. K. G. 1996, ApJS, 107, 747

Turner, B. E. 1989, ApJS, 70, 539 1991, ApJS, 76, 617

Willacy, K., \& Cherchneff, I. 1998, A\&A, 330, 676

Winters, J. M., Le Bertre, T., Nyman, L.-Å., Omont, A., \& Jeong, K. S. 2002, A\&A, 388, 609

Woods, P. M., Schöier, F. L., Nyman, L.-Å., \& Olofsson, H. 2003, A\&A, 402, 617

Yan, M., Federman, S. R., Dalgarno, A., \& Bjorkman, J. E. 1999, ApJ, 515, 640

Zhang, Y., Liu, X.-W., Luo, S.-G., Péquignot, D., \& Barlow, M. J. 2005, A\&A, 442, 249

Ziurys, L. M., Milam, S. N., Apponi, A. J., \& Woolf, N. J. 2007, Nature, 447, 1094 